\title{
AREAS OF $k$-DIMENSIONAL NONPARAMETRIC SURFACES IN $k+1$ SPACE
}

\author{
BY \\ ROBERT N. TOMPSON
}

Introduction. Suppose $X \subset E_{k}(k \geqq 2)$ is a $k$ cell, $g$ is a real-valued continuous function on $E_{k}, j$ is a positive integer not greater than $k$, and $f$ is the mapping defined by the relation

$$
f(x)=\left(x_{1}, \cdots, x_{j-1}, g(x), x_{j+1}, \cdots, x_{k}\right) \quad \text { for } x \in E_{k} .
$$

For this class of mappings on $E_{k}$ into $E_{k}$ (denoted by $\Omega_{k}$ ) we concern ourselves with the validity of the formula

$$
\int_{X}\left|D_{j} g(x)\right| d \mathcal{L}_{k} x=L(f \mid X)=\int_{E_{k}} S(f, X, y) d \mathcal{L}_{k} y=\int_{E_{k}} N(f, X, y) d \mathcal{L}_{k} y
$$

where $D_{j} g$ is the partial derivative of $g$ in the direction of the $j$ th base vector, $L(f \mid X)$ is the Lebesgue area of the surface $f \mid X, S(f, X, y)$ and $N(f, X, y)$ are respectively the stable multiplicity and multiplicity of $f$ on $X$, and $\mathcal{L}_{k}$ denotes the $k$-dimensional Lebesgue measure.

The main results, which comprise a complete theory of area for the class $\Omega_{k}$, are embodied in Theorems $2.6,2.13,2.14$, and 2.15.

For $k=2$ the results are in most part known (see [T1], [T2], and [R]).

The theory of area of the class $\Omega_{k}$ is intimately connected with the theory of area of $k$-dimensional nonparametric surfaces in $E_{k+1}$. In fact if $\bar{g}$ is the function defined by

$$
\bar{g}(x)=\left(x_{1}, x_{2}, \cdots, x_{k}, g(x)\right)
$$

for $x \in E_{k}$,

and $\pi$ is an orthogonal projection of $E_{k+1}$ onto $E_{k}$, then

$$
\bar{g} \mid X
$$

is a $k$-dimensional nonparametric surface in $E_{k+1}$, and

$$
\pi \circ \bar{g}
$$

is a mapping of $E_{k}$ into $E_{k}$. For some, but not all, orthogonal projections $\pi$, $\pi \circ \bar{g}$ is a member of $\Omega_{k}$.

A reduction procedure is devised whereby the theory of area of the class $\Omega_{k}$ extends, in great part, to the mappings $\pi \circ \bar{g}$. A theory of area for $k$-dimensional nonparametric surfaces in $E_{k+1}$ evolves; a theory in which complete information is obtained concerning the validity of the relation

Presented to the Society, June 20, 1954; received by the editors June 8, 1954. 


$$
\begin{aligned}
\int_{X} J \bar{g}(x) d \mathcal{L}_{k} x & =L(\bar{g} \mid X) \\
& =\beta(k+1, k)^{-1} \int_{G_{k+1}} \int_{E_{k}} S\left(p_{k+1}^{k} \circ R \circ \bar{g}, X, y\right) d \mathcal{L}_{k} y d \phi_{k+1} R \\
& =\beta(k+1, k)^{-1} \int_{G_{k+1}} \int_{E_{k}} N\left(p_{k+1}^{k} \circ R \circ \bar{g}, X, y\right) d \mathcal{L}_{k} y d \phi_{k+1} R
\end{aligned}
$$

where $J \bar{g}$ is the Jacobian associated with $\bar{g}$ by means of its approximate differential, and where the last two members of the string are respectively the stable integralgeometric and integralgeometric areas of the surface $\bar{g} \mid X$.

The main results are contained in Theorems 3.8, 3.11, 3.15, 3.16, and 3.17.

For $k=2$ the theory of area for $k$-dimensional nonparametric surfaces in $E_{k+1}$ is well established (see $[\mathrm{F} 4,6]$ and $[\mathrm{S}, \mathrm{V}]$ ).

1. Definitions.

1.1 Definition. If $f$ is a function, then inv $f$ is its inverse, and for any set $A, f \mid A$ is the function with domain ( $A \cap \operatorname{domain} f$ ) for which .

$$
(f \mid A)(x)=f(x) \quad \text { whenever } x \in(A \cap \text { domain } f) .
$$

Furthermore

$$
N(f, A, x)
$$

is the number (possibly $\infty$ ) of elements of the set $A \cap\{z \mid f(z)=x\}$, and

$$
f^{*}(A)=\{x \mid x=f(z) \text { for some } z \in A\} .
$$

If $g$ is also a function, then

$$
f \circ g \text {, }
$$

the superposition of $f$ on $g$, is defined by the formula

$$
(f \circ g)(x)=f(g(x))
$$

for all $x$.

1.2 Definition. Euclidean $n$ space will be denoted by $E_{n}$. The usual metric and inner product (denoted by $\bullet$ ) are assumed for this $n$-dimensional vector space. We write

$$
x=\left(x_{1}, x_{2}, \cdots, x_{n}\right) \quad \text { for } x \in E_{n} .
$$

For $m$ and $n$ positive integers we shall often identify $E_{m} \times E_{n}$ with $E_{m+n}$. Lebesgue $n$ dimensional measure over $E_{n}$ is denoted by $\mathcal{L}_{n}$.

$$
\alpha(n)=\mathcal{L}_{n}\left(E_{n} \cap\{x|| x \mid<1\}\right) .
$$

1.3 Definition. If $f$ is a function on $E_{n}$ to $E_{1}$ and $x \in E_{n}$, then we define $\lim _{z \rightarrow x} \sup \operatorname{ap} f(z)$ 
to be the infimum of the numbers of the form

$$
\lim _{z \rightarrow x} \sup _{z \in A} f(z)
$$

where $A$ is a Lebesgue measurable subset of $E_{n}$ with density one at $x$.

1.4 Definition. For $m$ and $n$ positive integers a function $L$ on $E_{m}$ to $E_{n}$ is linear if and only if

$$
\begin{array}{rlr}
L(x+y) & =L(x)+L(y) & \text { for } x \in E_{m}, y \in E_{m}, \\
L(\lambda x) & =\lambda L(x) & \text { for } x \in E_{m} \text { and } \lambda \text { a real number. }
\end{array}
$$

For $1 \leqq i \leqq m$ let ${ }^{m} I^{i}$ denote the $i$ th unit vector of $E_{m}$. Then if $L$ is a linear function on $E_{m}$ to $E_{n}$

$$
L\left(^{m} I^{i}\right)=L^{i}=\left(L_{1}^{i}, L_{2}^{i}, \cdots, L_{n}^{i}\right) \in E_{n} .
$$

The matrix of $L$, which we identify with $L$, is the $n$ by $m$ matrix whose entry in the $j$ th row and $i$ th column $(1 \leqq j \leqq n, 1 \leqq i \leqq m)$ is $L_{j}^{i}$. If $m \leqq n$ the square root of the sum of the squares of the determinants of all $m$ by $m$ minors will be denoted by $\Delta(L)$.

If $1 \leqq m \leqq n, f$ is a function on $E_{m}$ to $E_{n}$, and $L$ is a linear function on $E_{m}$ to $E_{n}$ for which

$$
\lim _{z \rightarrow x} \sup \operatorname{ap} \frac{|f(z)-f(x)-L(z-x)|}{|z-x|}=0,
$$

then $L$ is unique and is termed the approximate differential of $f$ at $x$. If $x$ is a point at which $f$ has the approximate differential $L$ we denote

$$
J f(x)=\Delta(L) .
$$

If $f$ is a function on $E_{1}$ into $E_{n}$ and $a<b$, then $T_{t=a}^{b} f(t)$ is the supremum of the numbers of the form

$$
\sum_{i=1}^{n}\left|f\left(t_{i}\right)-f\left(t_{i-1}\right)\right|
$$

where $a=t_{0} \leqq t_{1} \leqq \cdots \leqq t_{n}=b$.

If $f$ is a function on $E_{n}$ to $E_{1}$ and $j$ is a positive integer no greater than $n$, then $D_{j} f$ is the function on $E_{n}$ such that

$$
D_{i} f(x)=\lim _{n \rightarrow 0} \frac{f\left(x_{1}, \cdots, x_{j-1}, x_{j}+h, x_{j+1}, \cdots, x_{n}\right)-f(x)}{h} \quad \text { for } x \in E_{n} .
$$

1.5 Definition. If $m \leqq n$ are positive integers, then $p_{n}^{m}$ is the function on $E_{n}$ onto $E_{m}$ defined by

$$
p_{n}^{m}(x)=\left(x_{1}, \cdots, x_{m}\right) \quad \text { for } x \in E_{n} .
$$


1.6 Definition. If $n$ is a positive integer, then $G_{n}$ will denote the set of all linear transformations $R$ on $E_{n}$ to $E_{n}$ for which

$$
|R(x)|=|x| \quad \text { whenever } x \in E_{n} \text {. }
$$

With respect to the topology of uniform convergence and the operation of superposition, $G_{n}$ is a compact topological group, in fact, the orthogonal group of $E_{n}$.

The identity element of $G_{n}$ will be designated by ${ }^{n} I$, and $\phi_{n}$ will be the unique Haar measure over $G_{n}$ for which $\phi_{n}\left(G_{n}\right)=1$.

The following fact may be inferred (see $[\mathrm{W}, 8])$ :

If $f$ is a $\phi_{n}$ measurable function on $G_{n}$ then

$$
\int_{G_{n}} f(R) d \phi_{n} R=\int_{G_{n}} f(\operatorname{inv} R) d \phi_{n} R=\int_{G_{n}} f(R \circ S) d \phi_{n_{n}} R=\int_{G_{n}} f(S \circ R) d \phi_{n} R
$$

whenever $S \in G_{n}$.

1.7 Definition. If $m \leqq n$ are positive integers, then

$$
\beta(n, m)=\frac{\alpha(m) \cdot \alpha(n-m)}{\alpha(n) \cdot\left(\begin{array}{l}
n \\
m
\end{array}\right)} .
$$

1.8 Definition. A function $g$ on $E_{n}$ is said to be a gauge over $E_{n}$ if and only if domain $g \subset\left\{X \mid X \subset E_{n}\right\}$, range $g \subset\{t \mid 0 \leqq t \leqq \infty\}$. lation

If $g$ is a gauge over $E_{n}$ and $0<r \leqq \infty$, the function $\dot{g}_{r}$ is defined by the re-

$$
\ddot{g}_{r}(A)=\inf _{F \in B} \sum_{S \in F} g(S) \quad \text { for } A \subset E_{n},
$$

where $F \in B$ if and only if $F$ is a countable subfamily of domain $g$ for which

$$
A \subset \bigcup_{S \in F} S, \quad \text { diameter } S<r \text {, whenever } S \in F \text {. }
$$

One says that $\phi$ is generated by $g$ if and only if $g$ is a gauge over $E_{n}$ and $\phi$ is the function defined by

$$
\phi(A)=\lim _{r \rightarrow 0+} \dot{g}_{r}(A) \quad \text { for } A \subset E_{n} .
$$

It may be shown that $\phi$ is a (Carathéodory outer) measure over $E_{n}$ and that closed subsets of $E_{n}$ are $\phi$ measurable.

1.9 Definition. If $m \leqq n$ are positive integers and $\gamma_{n}^{m}, \zeta_{n}^{m}$, and $\chi_{n}^{m}$ are the gauges over $E_{n}$ defined by

$$
\gamma_{n}^{m}(S)=\sup _{R \in G} \mathcal{L}_{k}\left[\left(p_{n}^{m} \circ R\right) *(S)\right]
$$


whenever $S$ is a Borel subset of $E_{n}$,

$$
\zeta_{n}^{m}(S)=\beta(n, m)^{-1} \int_{G_{n}} \mathcal{L}_{k}\left[\left(p_{n}^{m} \circ R\right)^{*}(S)\right] d \phi_{n} R
$$

whenever $S$ is an analytic subset of $E_{n}$,

$$
\chi_{n}^{m}(S)=\alpha(m) 2^{-m}(\operatorname{diameter} S)^{m} \quad \text { whenever } S \subset E_{n},
$$

then $\mathcal{F}_{n}^{m}$ generated by $\chi_{n}^{m}, \mathcal{F}_{n}^{m}$ generated by $\zeta_{n}^{m}$, and $\Gamma_{n}^{m}$ generated by $\gamma_{n}^{m}$ are respectively the Hausdorff, the integralgeometric, and the Gross k-dimensional measures over $n$ space (see [F4], [H], [C]).

One may easily check that $\mathcal{F}_{n}^{m}, \mathcal{F}_{n}^{m}$, and $\Gamma_{n}^{m}$ are invariant under isometries of $E_{n}$ and that any subset of $E_{n}$ is contained in a $G_{\delta}$ set of equal $\mathcal{F C}_{n}^{m}$ measure, in an analytic set of equal $\mathcal{F}_{n}^{m}$ measure, and in a Borel set of equal $\Gamma_{n}^{m}$ measure. The equality of $\mathcal{F}_{n}^{n}$ and $\mathcal{L}_{n}$ is apparent from the definition. It is also true that $\mathfrak{F}_{n}^{n}=\mathcal{L}_{n}($ see $[\mathrm{SD}])$.

1.10 Definition. If $m \leqq n$ are positive integers and $X \subset E_{m}$ is an $m$ cell or its interior, then $C_{n}(X)$ will denote the set of continuous functions on $X$ to $E_{n}$.

If $g \in C_{n}(X)$, then $g$ is a polyhedron if and only if $X$ can be so triangulated that $g$ maps each simplex baracentrically onto a rectilinear simplex of $E_{n}$.

It is to be noted that relative to the topology of uniform convergence the class of polyhedra is dense in $C_{n}(X)$, and also that all areas used in this paper are equivalent on the class of polyhedra.

1.11 Definition. Suppose $m \leqq n$ are positive integers and $f$ is a continuous function on $E_{m}$ to $E_{n}$.

If $X \subset E_{m}$ is an $m$ cell, then

$$
L(f \mid X) \text {, the } m \text {-dimensional Lebesgue area of } f \mid X,
$$

is the lower limit of the areas of polyhedra approximating $f \mid X$.

If $X \subset E_{m}$ is the interior of an $m$ cell, then

$$
L(f \mid X) \text {, the } m \text {-dimensional Lebesgue area of } f \mid X,
$$

is the supremum of $L(f \mid Y)$ for all subsets $Y$ of $X$ which are $m$ cells.

If $X \subset E_{m}$ is either an $m$ cell or its interior, then

the m-dimensional Hausdorff area of $f \mid X=\int_{E_{n}} N(f, X, y) d \mathcal{H C}_{n}^{m} y$;

the m-dimensional integralgeometric area of $f \mid X=\int_{E_{n}} N(f, X, y) d \mathcal{F}_{n}^{m} y$

$$
=\beta(n, m)^{-1} \int_{G_{n}} \int_{E_{m}} N\left(p_{n}^{m} \circ R \circ f, X, x\right) d \mathcal{L}_{m} x d \phi_{n} R
$$


the $m$-dimensional integralgeometric stable area of $f \mid X$

$$
=\beta(n, m)^{-1} \int_{G_{n}} \int_{E_{m}} S\left(p_{n}^{m} \circ R \circ f, X, x\right) d \mathcal{L}_{m} x d \phi_{n} R ;
$$

where $S(f, X, x)$ is the stable multiplicity as defined in [F7].

1.12 Definition. If $n$ is a positive integer exceeding one, $j$ is a positive integer no greater than $n, A \subset E_{n}, z \in E_{n}$, and $u \in E_{n-1}$, then

$$
\begin{aligned}
A_{z} & =E_{n} \cap\{x+z \mid x \in A\}, \\
A_{(j)} & =E_{n-1} \cap\left\{w \mid\left(w_{1}, \cdots, w_{j-1}, v, w_{j+1}, \cdots, w_{n}\right) \in A \text { for some } v\right\}, \\
A_{(j)}^{u} & =E_{1} \cap\left\{v \mid\left(u_{1}, \cdots, u_{j-1}, v, u_{j+1}, \cdots, u_{n}\right) \in A\right\} .
\end{aligned}
$$
then

If $f$ is a continuous real-valued function on $E_{n}$ and $i$ is a positive integer,

$$
K_{i}=E_{n} \cap\left\{x|| x \mid \leqq i^{-1}\right\},
$$

and $f_{i}$, the $i$ th integral mean, of $f$, is the real-valued function on $E_{n}$ defined by the formula

$$
f_{i}(x)=\alpha(n)^{-1} i^{n} \int_{K i} f(x+z) d \mathcal{L}_{n} z \quad \text { for } x \in E_{n} .
$$

2. On a certain class of mappings of $E_{k}$ into $E_{k}(k>1)$. Let $\Omega_{k}$ denote the class of mappings on $E_{k}$ into itself defined by: $f \in \Omega_{k}$ if and only if for some positive integer $j$ not exceeding $k$ and for some continuous real-valued function $g$ on $E_{k}, f$ is defined by the formula

$$
f(x)=\left(x_{1}, \cdots, x_{j-1}, g(x), x_{j+1}, \cdots, x_{k}\right) \quad \text { for } x \in E_{k} .
$$

For such a function $f \in \Omega_{k}$ and $i$ a positive integer, $\dot{f}_{i}$ will be defined as that element of $\Omega_{k}$ for which

$$
\dot{f}_{i}(x)=\left(x_{1}, \cdots, x_{i-1}, g_{i}(x), x_{j+1}, \cdots, x_{k}\right) \quad \text { for } x \in E_{k} .
$$

2.1 Sectional assumption. In the development of a theory of area for the class $\Omega_{k}$ it will be convenient to fix a function $f \in \Omega_{k}$ which will be defined by $f(x)=\left(x_{1}, \cdots, x_{k-1}, g(x)\right)$ for $x \in E_{k}$, where $g$ is a (fixed) real-valued continuous function on $E_{k}$.

No restriction in generality will be effected by this assumption (see Remark 2.20). then

2.2 Lemma. If $Y \subset E_{k}$ is a bounded open convex set and $i$ is a positive integer,

$$
\int_{E_{k}} N\left(\dot{f}_{i}, Y, x\right) d \mathcal{L}_{k} x \leqq \alpha(k)^{-1} i^{k} \int_{\boldsymbol{K}_{i}} \int_{\boldsymbol{E}_{\boldsymbol{k}}} N\left(f, Y_{z}, x\right) d \mathcal{L}_{k} x d \mathcal{L}_{k} z
$$


Proof. We let

$$
a(u)=\inf Y_{(k)}^{u}, \quad b(u)=\sup Y_{(k)}^{u} \quad \text { for } u \in Y_{(k)},
$$

and for each $u \in Y_{(k)}$ we define the function

$$
\begin{aligned}
& h_{u}: \operatorname{closure} Y_{(k)}^{u} \rightarrow E_{1}, \\
& h_{u}(v)=g\left(u_{1}, u_{2}, \cdots, u_{k-1}, v\right) \quad \text { for } v \in \operatorname{closure} Y_{(k)}^{u} .
\end{aligned}
$$

The remainder of the proof is divided into four parts.

PART 1. $N(f, Y,(u, v))=N\left(h_{u}, Y_{(k)}^{u}, v\right)$ for $(u, v) \in E_{k-1} \times E_{1}$.

Proof. If $A$ is a subset of $E_{k}$ we shall denote by $q(A)$ the number (possibly $\infty$ ) of elements of $A$. Whence

$$
\begin{aligned}
N(f, Y,(u, v)) & =q\left[\left(E_{k-1} \times E_{1}\right) \cap\{(w, t) \mid f(w, t)=(u, v)\}\right] \\
& =q\left[\left(E_{k-1} \times E_{1}\right) \cap\left\{(w, t) \mid w=u, t \in Y_{(k)}^{w}, h_{v}(t)=v\right\}\right] \\
& =q\left[E_{1} \cap\left\{t \mid t \in Y_{(k)}^{u}, h_{u}(t)=v\right\}\right] \\
& =N\left(h_{u}, Y_{(k)}^{u}, v\right) .
\end{aligned}
$$

PART 2. $\int_{E_{k}} N(f, Y,(u, v)) d \mathcal{L}_{k}(u, v)=\int_{\left.Y_{k}\right)} T_{v=a(u)}^{o(u)} g(u, v) d \mathcal{L}_{k-1} u$.

Proof. Using Part 1 and $[\mathrm{F} 1,4.3]$ we compute:

$$
\begin{aligned}
\int_{E_{k}} N(f, Y,(u, v)) d \mathcal{L}_{k}(u, v) & =\int_{E_{k-1}} \int_{B_{1}} N(f, Y,(u, v)) d \mathcal{L}_{1} v d \mathcal{L}_{k-1} u \\
& =\int_{Y_{(k)}} \int_{E_{1}} N\left(h_{u}, Y_{(k)}^{u}, v\right) d \mathcal{L}_{1} v d \mathcal{L}_{k-1} u \\
& =\int_{Y_{(k)}} \int_{E_{1}} N\left(h_{u}, \text { closure } Y_{(k)}^{u}, v\right) d \mathcal{L}_{1} v d \mathcal{L}_{k-1} u \\
& =\int_{Y_{(k)}} T_{v=a(u)}^{b(u)} h_{u}(v) d \mathcal{L}_{k-1} u \\
& =\int_{Y_{(k)}} T_{v=a(u)}^{b(u)} g(u, v) d \mathcal{L}_{k-1} u .
\end{aligned}
$$

PART 3. If $c$ and $d$ are real numbers with

$$
c<d \text { and } z=\left(z^{\prime}, z^{\prime \prime}\right) \in E_{k-1} \times E_{1},
$$

then

$$
T_{v=c}^{d} g_{i}(u, v) \leqq \alpha(k)^{-1} i^{k} \int_{K_{i}} T_{v=c}^{d} g\left(u+z^{\prime}, v+z^{\prime \prime}\right) d \mathcal{L}_{k} z .
$$

Proof. Let $c=v_{0}<v_{1}<\cdots<v_{m}=d$. Then 


$$
\begin{aligned}
\sum_{p=1}^{m} \mid g_{i}(u, & \left.v_{p}\right)-g_{i}\left(u, v_{p-1}\right) \mid \\
& =\sum_{p=1}^{m}\left|\alpha(k)^{-1} i^{k} \int_{K_{i}}\left\{g\left(u+z^{\prime}, v_{p}+z^{\prime \prime}\right)-g\left(u+z^{\prime}, v_{p-1}+z^{\prime \prime}\right)\right\} d \mathcal{L}_{k} z\right| \\
& \leqq \alpha(k)^{-1} i^{k} \int_{K_{i}} \sum_{p=1}^{m}\left|g\left(u+z^{\prime}, v_{p}+z^{\prime \prime}\right)-g\left(u+z^{\prime}, v_{p-1}+z^{\prime \prime}\right)\right| d \mathcal{L}_{k} z \\
& \leqq \alpha(k)^{-1} i^{k} \int_{K_{i}} T_{v=c}^{d} g\left(u+z^{\prime}, v+z^{\prime \prime}\right) d \mathcal{L}_{k} z .
\end{aligned}
$$

PART 4. $\int_{E_{k}} N\left(\dot{f_{i}}, Y, x\right) d \mathcal{L}_{k} x \leqq \alpha(k)^{-1} i^{k} \int_{K_{i}} \int_{E_{k}} N\left(f, Y_{z}, x\right) d \mathcal{L}_{k} x d \mathcal{L}_{k} z$.

Proof. Suppose $z=\left(z^{\prime}, z^{\prime \prime}\right) \in E_{k-1} \times E_{1}$. We know that

$$
Y=\left(E_{k-1} \times E_{1}\right) \cap\left\{(u, v) \mid v \in Y_{(k)}^{u}\right\} .
$$

It is easy to check that

$$
Y_{z}=\left(E_{k-1} \times E_{1}\right) \cap\left\{(u, v) \mid v-z^{\prime \prime} \in Y_{(k)}^{u-z^{\prime}}\right\}, \quad\left[Y_{(k)}\right]_{z^{\prime}}=\left[Y_{z}\right]_{(k)} .
$$

Letting $x=(u, v) \in E_{k-1} \times E_{1}$ and applying Part 2 to $\dot{f}_{i}$ we infer with the help of Parts 3 and 2 that

$$
\begin{aligned}
\int_{E_{k}} N\left(\dot{f}_{i}, Y, x\right) d \mathcal{L}_{k} x & =\int_{Y(k)} T_{v=a(u)}^{b(u)} g_{i}(u, v) d \mathcal{L}_{k-1} u \\
& \leqq \int_{Y(k)} \alpha(k)^{-1} i^{b} \int_{K_{i}} T_{v=a(u)}^{b(u)} g\left(u+z^{\prime}, v+z^{\prime \prime}\right) d \mathcal{L}_{k} z d \mathcal{L}_{k-1} u \\
& =\alpha(k)^{-1} i^{k} \int_{K_{i}} \int_{Y_{(k)}} T_{v=a(u)}^{b(u)} g\left(u+z^{\prime}, v+z^{\prime \prime}\right) d \mathcal{L}_{k-1} u d \mathcal{L}_{k} z \\
& =\alpha(k)^{-1} i^{k} \int_{K_{i}} \int_{[Y(k)] z^{\prime}} T_{v=a\left(u-z^{\prime}\right)}^{b\left(u-z^{\prime}\right)} g\left(u, v+z^{\prime \prime}\right) d \mathcal{L}_{k-1} u d \mathcal{L}_{k} z \\
& =\alpha(k)^{-1} i^{k} \int_{K_{i}} \int_{\left[Y_{S}\right](k)} T_{v=a\left(u-z^{\prime}\right)+z^{\prime \prime}}^{b\left(u(u, v) d \mathcal{L}_{k-1} u d \mathcal{L}_{k} z\right.} \\
& =\alpha(k)^{-1} i^{k} \int_{K_{i}} \int_{E_{k}} N\left(f, Y_{z}, x\right) d \mathcal{L}_{k} x d \mathcal{L}_{k} z .
\end{aligned}
$$

2.3 Lemma. If $X \subset E_{k}$ is a $k$ cell with boundary $\dot{X}, F \in C_{k}(X), G \in C_{k}(X)$, and if $F \mid \dot{X}$ is homotopic to $G \mid \dot{X}$ where

$$
\begin{aligned}
& F \mid \dot{X} \text { is a map of } \dot{X} \text { into } E_{k}-\{y\}, \\
& G \mid \dot{X} \text { is an essential map of } \dot{X} \text { into } E_{k}-\{y\},
\end{aligned}
$$

then $y$ is a stable value of $F \mid X-\dot{X}$. 
Proof. Let $r=\inf _{x \in \dot{X}}|F(x)-y|$, and let $\pi$ denote the function on $E_{k}-\{y\}$ into the $k-1$ sphere defined by the formula

$$
\pi(x)=\frac{x-y}{|x-y|} \quad \text { for } x \in E_{k}-\{y\} .
$$

The proof will be divided into three parts.

PART 1. If degree $(\pi \circ F \mid \dot{X}) \neq 0$, then there exists $a p \in X-\dot{X}$ for which $F(p)=y$.

Proof. Since $X$ is a $k$ cell, for $q \in X$ we may define a continuous contraction $\phi$ of $X$ into $\{q\}$ :

$$
\begin{array}{lr}
\phi:\{t \mid 0 \leqq t \leqq 1\} \rightarrow\{H \mid H \text { is a continuous function on } X \text { into } X\}, \\
(\phi(0))(x)=x & \text { for } x \in X, \\
(\phi(1))(x)=q & \text { for } x \in X .
\end{array}
$$

Suppose that the statement is false. Then for $0 \leqq t \leqq 1$

$$
\begin{aligned}
& F \circ \phi(t) \mid \dot{X}: \dot{X} \rightarrow E_{k}-\{y\}, \\
& F \circ \phi(t) \mid \dot{X} \text { is homotopic to } F \circ \phi(0) \mid \dot{X}, \\
& \pi \circ F \circ \phi(t) \mid \dot{X} \text { is homotopic to } \pi \circ F \circ \phi(0) \mid \dot{X} .
\end{aligned}
$$

Accordingly we arrive at the contradiction $0=$ degree $(\pi \circ F \circ \phi(1) \mid \dot{X})=\operatorname{degree}(\pi \circ F \circ \phi(0) \mid \dot{X})=\operatorname{degree}(\pi \circ F \mid \dot{X}) \neq 0$.

PART 2. If $H \in C_{k}(X)$ and $|H| \dot{X}-F|\dot{X}|<r$, then

$$
\text { degree }(\pi \circ H \mid \dot{X})=\text { degree }(\pi \circ F \mid \dot{X}) \text {. }
$$

Proof. Since for $x \in \dot{X}$

$$
|F(x)-H(x)|<|F(x)-y|
$$

it follows that

$$
y \notin E_{k} \cap\{z \mid z=F(x)+t(H(x)-F(x)) \text { for } 0 \leqq t \leqq 1\} .
$$

Hence it suffices to define the continuous function

$$
\begin{aligned}
& \phi: \dot{X} \times\{t \mid 0 \leqq t \leqq 1\} \rightarrow E_{k}-\{y\}, \\
& \phi(x, t)=F(x)+t(H(x)-F(x)) \quad \text { for }(x, t) \in \dot{X} \times\{t \mid 0 \leqq t \leqq 1\},
\end{aligned}
$$

to establish that $F \mid \dot{X}$ and $H \mid \dot{X}$ are homotopic. Consequently $\pi \circ F \mid \dot{X}$ and $\pi \circ H \mid \dot{X}$ are homotopic and

$$
\text { degree }(\pi \circ F \mid \dot{X})=\text { degree }(\pi \circ H \mid \dot{X}) \text {. }
$$

PART 3. $y$ is a stable value of $F$.

Proof. We infer from the hypothesis that 


$$
\text { degree }(\pi \circ F \mid \dot{X})=\text { degree }(\pi \circ G \mid \dot{X}) \neq 0 \text {. }
$$

If $L \in C_{k}(X)$ and $|L-F|<r$, we may use Part 2 to establish that

$$
\text { degree }(\pi \circ L \mid \dot{X})=\text { degree }(\pi \circ F \mid \dot{X}) \text {, }
$$

and Part 1 to show the existence of a $p \in X-\dot{X}$ for which $L(p)=y$.

2.4 Theorem. If $Y$ is a bounded open convex subset of $E_{k}$, then

$$
N(f, Y, x)=S(f, Y, x) \quad \text { for } \mathcal{L}_{k} \text { almost all } x \in E_{k} .
$$

Proof. We assume $h_{u}$ to have the same meaning as in Lemma 2.2. Let $C$ be the set of points $(u, v)$ in $Y$ for which $h_{u}$ has either a relative maximum or a relative minimum at $v$. We may check that $C$ is a $F_{\sigma}$ set and

$$
h_{u}^{*}\left(C_{(k)}^{u}\right) \text { is countable for } u \in Y_{(k)} .
$$

If follows that $f^{*}(C)$ is $\mathcal{L}_{k}$ measurable and

$$
\mathcal{L}_{k}\left[f^{*}(C)\right]=0 .
$$

We know that

$$
\begin{aligned}
N(f, Y-C, x) & =N(f, Y, x) & & \text { for } x \in f^{*}(Y-C)-f^{*}(C), \\
N(f, Y, x) & =S(f, Y, x) & & \text { for } x \in f^{*}(Y),
\end{aligned}
$$

hence we may complete the proof by showing that $f$ is stable $[\mathrm{F} 4,6.6]$ at every point of $Y-C$. For if this were so, then

$$
S(f, Y, x) \leqq N(f, Y, x)=N(f, Y-C, x) \leqq S(f, Y, x)
$$

whenever $x \in f^{*}(Y-C)-f^{*}(C)$.

Let $\epsilon>0$.

If $\left(u^{0}, v^{0}\right)=\left(u_{1}^{0}, u_{2}^{0}, \cdots, u_{k-1}^{0}, v^{0}\right) \in Y-C$, then using the continuity of $g$ we can select

$$
u^{1} \in Y_{(k)}, \quad u^{2} \in Y_{(k)}, \quad v^{1} \in E_{1}, \quad v^{2} \in E_{1},
$$

satisfying

$$
\begin{aligned}
& u_{i}^{0}-\epsilon<u_{i}^{1}<u_{i}^{0}<u_{i}^{2}<u_{i}^{0}+\epsilon \\
& v^{0}-\epsilon<v^{1}<v^{0}<v^{2}<v^{0}+\epsilon,
\end{aligned} \quad \text { for } i=1,2, \cdots, k-1,
$$

and such that if we denote

$$
\begin{aligned}
& P=E_{k-1} \cap\left\{u \mid u_{i}^{1} \leqq u_{i} \leqq u_{i}^{2} \text { for } i=1,2, \cdots, k-1\right\}, \\
& X=\left(E_{k-1} \times E_{1}\right) \cap\left\{(u, v) \mid u \in P \text { and } v^{1} \leqq v \leqq v^{2}\right\},
\end{aligned}
$$

then $X \subset Y$ and either

$$
h_{u}\left(v^{1}\right)<h_{u}\left(v^{0}\right)<h_{u}\left(v^{2}\right) \quad \text { whenever } u \in P,
$$


or else

$$
h_{u}\left(v^{1}\right)>h_{u}\left(v^{0}\right)>h_{u}\left(v^{2}\right) \quad \text { whenever } u \in P .
$$

Observe that $X$ is a $k$ cell with diameter less than $2 k^{1 / 2} \epsilon$ and, denoting its boundary by $\dot{X}$ and $f\left(u^{0}, v^{0}\right)$ by $y$, that $f \mid \dot{X}$ is a map of $\dot{X}$ into $E_{k}-\{y\}$.

If (1) occurs we can define the function $G$ on $\dot{X}$ into $E_{k}-\{y\}$ by

$$
G(u, v)=\left(u, y_{k}+v-v^{0}\right) \quad \text { for }(u, v) \in\left(E_{k-1} \times E_{1}\right) \cap \dot{X},
$$

and the function $\phi$ on $\dot{X} \times\{t \mid 0 \leqq t \leqq 1\}$ into $E_{k}-\{y\}$ by

$$
\phi(u, v, t)=\left(u,(1-t)\left(y_{k}+v-v^{0}\right)+t h_{u}(v)\right)
$$

for $(u, v, t) \in\left(\left(E_{k} \times E_{1}\right) \cap \dot{X}\right) \times\{t \mid 0 \leqq t \leqq 1\}$, and infer that

$G$ is an essential map of $\dot{X}$ into $E_{k}-\{y\}$,

$G$ is homotopic to $f \mid \dot{X}$.

Lemma 2.3 implies that $y$ is a stable value of $f \mid X-\dot{X}$, and from the arbitrary nature of $\epsilon$ we conclude that $f$ is stable at $\left(u^{0}, v^{0}\right)$.

Whenever (2) occurs a similar treatment is employed. The proof is complete.

2.5 Lemma. If $X \subset E_{k}$ is a $k$ cell with boundary $\dot{X}$, then $\mathcal{L}_{k}\left[f^{*}(\dot{X})\right]=0$.

Proof. Let

$$
h(x)=\left(x_{1}, x_{2}, \cdots, x_{k}, g(x)\right) \quad \text { for } x \in X .
$$

Then observing that $\dot{X}$ lies on $2 k k-1$ cells $A_{i}(i=1,2, \cdots, 2 k)$ it is apparent that the $h$ image of each such $k-1$ cell lies on a $k$ plane in $E_{k+1}$, and that

$$
\mathcal{H}_{k+1}^{k}\left[h^{*}(\dot{X})\right] \leqq \sum_{i=1}^{2 k} \mathcal{H C}_{k+1}^{k}\left[h^{*}\left(A_{i}\right)\right] .
$$

If $j$ is a positive integer no greater than $2 k$, let $\pi$ be an isometric projection onto $E_{k}$ of the $k$ plane containing $A_{j}$ and $h^{*}\left(A_{j}\right)$, which satisfies

$$
\pi^{*}\left(A_{j}\right) \subset E_{k} \cap\left\{x \mid x_{k}=0\right\} \text {. }
$$

It follows that

$$
\text { the number of elements in }\left[(\pi \circ h)^{*}\left(A_{j}\right)\right]_{(k)}^{u}=1
$$

for $u \in\left[(\pi \circ h)^{*}\left(A_{j}\right)\right]_{(k)}$. We apply Fubini's theorem to obtain

$$
\begin{aligned}
\operatorname{FC}_{k+1}^{k}\left[h^{*}\left(A_{j}\right)\right] & =\mathcal{L}_{k}\left[(\pi \circ h)^{*}\left(A_{j}\right)\right] \\
& =\int_{\left[\left(\pi_{0} h\right)^{*}(A j)\right](k)} \mathcal{L}_{1}\left\{\left[(\pi \circ h)^{*}\left(A_{j}\right)\right]_{(k)}^{u}\right\} d \mathcal{L}_{k-\mathrm{i}} u=0, \\
\operatorname{FC}_{k+1}^{k}\left[h^{*}(\dot{X})\right] & =0 .
\end{aligned}
$$


Finally letting $R$ be the element of $G_{k}$ for which

$$
R(w)=\left(w_{1}, \cdots, w_{k-1}, w_{k+1}, w_{k}\right) \quad \text { for } w \in E_{k+1},
$$

we conclude from the Lipschitzian charácter of $p_{\boldsymbol{k}+1}^{k} \circ R$ that

$$
\mathcal{L}_{k}\left[f^{*}(\dot{X})\right]=\mathcal{L}_{k}\left[\left(p_{k+1}^{k} \circ R \circ h\right)^{*}(\dot{X})\right]=0 .
$$

2.6 THEOREM. If $X \subset E_{k}$ is a $k$ cell, then

$$
\int_{\boldsymbol{E}_{\boldsymbol{k}}} N(f, X, x) d \mathcal{L}_{k} x=\int_{\boldsymbol{E}_{\boldsymbol{k}}} S(f, X, x) d \mathcal{L}_{k} x=L(f \mid X)=\lim _{\boldsymbol{\jmath} \rightarrow \infty} L\left(\dot{f}_{j} \mid X\right) .
$$

Proof. Let $Y$ denote the interior of $X$ and let $A$ be an open interval of $E_{k}$ for which

$$
\text { closure } A \subset Y \text {. }
$$

Select a positive integer $i$ so large that

$$
A_{z} \subset Y
$$

for $z \in K_{i}$.

Then using $[F 4,6.13],[F 2,4.5]$ and Lemma 2.2 we compute

$$
\begin{aligned}
L\left(\dot{f_{i}} \mid \text { closure } A\right) & =\int_{A} J \dot{f}_{i}(x) d \mathcal{L}_{k} x \\
& =\int_{E_{k}} N\left(\dot{f}_{i}, A, x\right) d \mathcal{L}_{k} x \\
& \leqq \alpha(k)^{-1} i^{k} \int_{\boldsymbol{K}_{i}} \int_{E_{k}} N\left(f, A_{z}, x\right) d \mathcal{L}_{k} x d \mathcal{L}_{k} z \\
& \leqq \int_{E_{k}} N(f, Y, x) d \mathcal{L}_{k} x .
\end{aligned}
$$

It follows using the lower semi-continuity of $L$ on $C_{k}(X)$ that

$$
L(f \mid \text { closure } A) \leqq \liminf _{i \rightarrow \infty} L\left(\dot{f}_{i} \mid \text { closure } A\right) \leqq \int_{E_{k}} N(f, Y, x) d \mathcal{L}_{k} x .
$$

From the arbitrary nature of $A$ and Theorem 2.4 we may conclude

$$
L(f \mid Y) \leqq \int_{\boldsymbol{E}_{\boldsymbol{k}}} N(f, Y, x) d \mathcal{L}_{k} x=\int_{\boldsymbol{E}_{\boldsymbol{k}}} S(f, Y, x) d \mathcal{L}_{k} x \leqq L(f \mid Y) .
$$

In view of Lemma 2.5 it is seen that this relation holds with $Y$ replaced by $X$.

For the other part of the statement we recall in general that

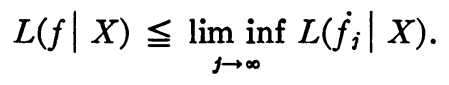


Therefore under the assumption that $L(f \mid X)$ is finite we need to prove that

$$
\limsup _{j \rightarrow \infty} L\left(\dot{f}_{i} \mid X\right) \leqq L(f \mid X) \text {. }
$$

Pick $\epsilon>0$. Utilizing the foregoing results we can select an open interval $U$ of $E_{k}$ such that

$$
X \subset U, \quad L(f \mid U) \leqq L(f \mid X)+\epsilon .
$$

Let $j$ be so large a positive integer that

$$
\text { (interior } X)_{z} \subset U
$$

whenever $z \in K_{j}$.

Then we can show just as before that

$$
L\left(\dot{f}_{j} \mid X\right) \leqq \int_{E_{k}} S(f, U, x) d \mathcal{L}_{k} x \leqq L(f \mid U) \leqq L(f \mid X)+\epsilon .
$$

Accordingly

$$
\limsup _{j \rightarrow \infty} L\left(\dot{f}_{j} \mid X\right) \leqq L(f \mid X)+\epsilon .
$$

Since $\epsilon$ was arbitrary this completes the proof.

2.7 REMARK. Using the results of the preceding theorem and the notation of [F 6] we find for $X \subset E_{k}$ a $k$ cell that

$$
\begin{aligned}
L(f \mid X) & =M^{* *}(f \mid X)=M^{*}(f \mid X)=S^{* *}(f \mid X)=S^{*}(f \mid X) \\
& =V^{* *}(f \mid X)=V^{*}(f \mid X)=U^{* *}(f \mid X)=U^{*}(f \mid X) \\
& =N^{* *}(f \mid X)=N^{*}(f \mid X) .
\end{aligned}
$$

2.8 Definition. If $X \subset E_{k}$ is a $k$ cell and $h \in C_{1}(X)$, then

(i) for $i$ a positive integer no greater than $k, h$ is said to be of bounded variation ( $i$ ) in the sense of Tonelli (BVT $(i)$ ) on $X$ if and only if

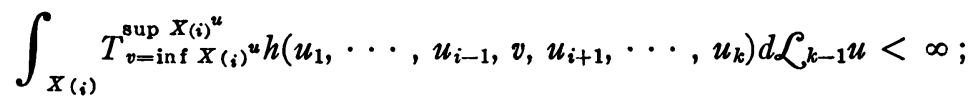

(ii) $h$ is said to be of bounded variation in the sense of Tonelli (BVT) on $X$ if and only if $h$ is BVT $(i)$ on $X$ whenever $i=1,2, \cdots, k$.

2.9 Definition. If $X \subset E_{k}$ is a $k$ cell and $h \in C_{1}(X)$, then

(i) for $i$ a positive integer no greater than $k, h$ is said to be absolutely continuous (i) in the sense of Tonelli (ACT (i)) on $X$ if and only if $h$ is BVT (i) on $X$ and the function

$$
v \rightarrow h\left(u_{1}, \cdots, u_{i-1}, v, u_{i+1}, \cdots, u_{k}\right)
$$

is absolutely continuous in the classical sense on $X_{(i)}^{u}$, for $\mathcal{L}_{k-1}$ almost all $u \in X_{(i)}$; 
(ii) $h$ is said to be absolutely continuous in the sense of Tonelli (ACT) on $X$ if and only if $h$ is ACT $(i)$ on $X$ whenever $i=1,2, \cdots, k$.

2.10 Definition. If $X \subset E_{k}$ is a $k$ cell and $h \in C_{k}(X)$, then $h$ is said to be absolutely continuous on $X$ if and only if

$$
\int_{E_{k}} N(h, X, y) d \mathcal{L}_{k} y<\infty,
$$

and $h$ transforms subsets of $X$ of $\mathcal{L}_{k}$ measure zero into sets of $\mathcal{L}_{k}$ measure zero.

2.11 Sectional assumption. For the rest of this section $X \subset E_{k}$ will denote a $k$ cell.

2.12 Lemma. If $g$ is $B V T(k)$ on $X$, then

$$
\begin{aligned}
\int_{Y}\left|D_{k} g(x)\right| d \mathcal{L}_{k} x & =\int_{Y_{(k)}} \int_{Y_{(k)}{ }^{u}}\left|D_{k} g(u, v)\right| d \mathcal{L}_{1} v d \mathcal{L}_{k-1} u \\
& \leqq \int_{Y_{(k)}} T_{\left.v=\text { inf } Y_{(k)}\right)^{u}}^{\sup Y_{(k}} g(u, v) d \mathcal{L}_{k-1} u \\
& =\int_{E_{k}} N(f, Y,(u, v)) d \mathcal{L}_{k}(u, v) \\
& =\int_{E_{k}} N(f, Y, y) d \mathcal{L}_{k} y
\end{aligned}
$$

whenever $Y \subset X$ and $Y$ is a $k$ cell.

Proof. This statement follows directly from Definition 2.8 and Part 2 of Lemma 2.2.

2.13 ThEOREM. The following statements are equivalent:

(i) $g$ is $B V T(k)$ on $X$,

(ii) $L(f \mid X)<\infty$.

Proof. By virtue of Part 2 of Lemma 2.2 and Theorem 2.6

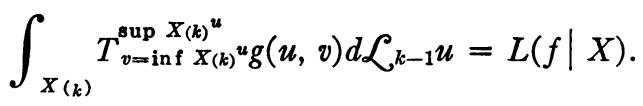

The theorem is an immediate consequence of this equality.

2.14 Theorem. If $g$ is $B V T(k)$ on $X, U$ is the set of those points $x$ in $X$ for which $D_{k} g(x)$ exists, $Y$ is an $\mathcal{L}_{k}$ measurable subset of $X$ and $Z$ is an analytic subset of $E_{k}$ contained in $X$, then

(i) $U$ is a Borel subset of $E_{k}$ and $\mathcal{L}_{k}(X-U)=0$,

(ii) $\int_{Y}\left|D_{k} g(x)\right| d \mathcal{L}_{k} x=\int_{E_{k}} N(f, Y \cap U, y) d \mathcal{L}_{k} y<\infty$,

(iii) $\int_{Z}\left|D_{k} g(x)\right| d \mathcal{L}_{k} x \leqq \int_{E_{k}} N(f, Z, y) d \mathcal{L}_{k} y<\infty$. 
Proof. We know from [S, V4.1] that $D_{k} g$ is a Borel measurable function. We may infer that

$U$ is a Borel subset of $E_{k}$,

and since

$$
\mathcal{L}_{1}\left[(X-U)_{(k)}^{u}\right]=0 \quad \text { for } \mathcal{L}_{k-1} \text { almost all } u \in X_{(k)},
$$

may use Fubini's theorem to conclude that

$$
\mathcal{L}_{k}(X-U)=0 \text {. }
$$

Using the notation of Lemma 2.2 the statement

$$
\left|D_{k} g(u, v)\right|=\left|D_{1} h_{u}(v)\right|=J h_{u}(v) \quad \text { for } v \in U_{(k)}^{u}
$$

is true for $u \in X_{(k)}$. Also we know that

$$
(Y \cap U)_{(k)}^{u}
$$

is an $\mathcal{L}_{1}$ measurable subset of $E_{1}$ for $u \in X_{(k)}$.

Upon combining these facts and using $[\mathrm{F} 2,5.2]$ (applied to $h_{u}$ ), Part 1 of Lemma 2.2, and Theorem 2.13 , we find that

$$
\begin{aligned}
\int_{Y}\left|D_{k} g(x)\right| d \mathcal{L}_{k} x & =\int_{Y \cap U}\left|D_{k} g(x)\right| d \mathcal{L}_{k} x \\
& =\int_{(Y \cap U)_{(k)}} \int_{(Y \cap U)_{(k)}}\left|D_{k} g(u, v)\right| d \mathcal{L}_{{ }_{1}} v d \mathcal{L}_{k-1} u \\
& =\int_{(Y \cap U)_{(k)}} \int_{(Y \cap U)_{(k)}{ }^{u}} J h_{u}(v) d \mathcal{L}_{1} v d \mathcal{L}_{k-1} u \\
& =\int_{(Y \cap U)_{(k)}} \int_{E_{1}} N\left(h_{u},(Y \cap U)_{(k)}^{u}, v\right) d \mathcal{L}_{{ }_{1}} v d \mathcal{L}_{k-1} u \\
& =\int_{E_{k-1}} \int_{E_{1}} N(f, Y \cap U,(u, v)) d \mathcal{L}_{1} v d \mathcal{L}_{k-1} u \\
& =\int_{E_{k}} N(f, Y \cap U, y) d \mathcal{L}_{k} y<\infty .
\end{aligned}
$$

For (iii) it is only necessary in addition to observe that

$$
\begin{gathered}
N(f, Z, y) \text { is } \mathcal{L}_{k} \text { measurable in } y, \\
\int_{\boldsymbol{E}_{\boldsymbol{k}}} N(f, Z, y) d \mathcal{L}_{k} y<\infty .
\end{gathered}
$$

2.15 THEOREM. If $\mathrm{g}$ is $B V T(k)$ on $X$, then the following statements are equivalent: 
(i) $f$ is absolutely continuous on $X$,

(ii) $\int_{Y}\left|D_{k} g(x)\right| d \mathcal{L}_{k} x=\int_{E_{k}} N(f, Y, y) d \mathcal{L}_{k} y<\infty$ whenever $Y$ is an $\mathcal{L}_{k}$ measurable subset of $X$,

(iii) $\int_{X}\left|D_{k} g(x)\right| d \mathcal{L}_{k} x=\int_{E_{k}} N(f, X, y) d \mathcal{L}_{k} y<\infty$,

(iv) $g$ is $A C T(k)$ on $X$.

Proof. The proof is divided into five parts.

PART 1. (i) implies (ii).

Proof. Let $U$ be the set of points $x$ in $X$ for which $D_{k} g(x)$ exists, and $Y$ be an $\mathcal{L}_{k}$ measurable subset of $X$. Then

$$
\begin{gathered}
N(f, Y, y) \text { is } \mathcal{L}_{k} \text { measurable in } v, \\
\mathcal{L}_{k}(Y-U)=0,
\end{gathered}
$$

and we may infer, using the preceding theorem, that

$$
\begin{aligned}
\int_{Y}\left|D_{k} g(x)\right| d \mathcal{L}_{k} x & =\int_{E_{k}} N(f, Y \cap U, y) d \mathcal{L}_{k} y \\
& =\int_{E_{k}} N(f, Y, y) d \mathcal{L}_{k} y<\infty .
\end{aligned}
$$

PART 2. (ii) implies (iii).

PART 3. (iii) implies (iv).

Proof. Lemma 2.12 implies that

$$
T_{v=\text { inf } X(k)}^{\sup X(k)} u^{u} g(u, v)=\int_{\left.X_{(k)}\right)^{u}}\left|D_{k} g(u, v)\right| d \alpha, 1^{v}
$$

for $\mathcal{L}_{k-1}$ almost all $u \in X_{(k)}$. Whence

$$
g \text { is } A C T(k) \text { on } X \text {. }
$$

PART 4. (iv) implies (ii).

Proof. If $Y \subset X$ and $Y$ is a $k$ cell, then (iv) being true means

$$
T_{v=\inf Y_{(k)}{ }^{u} g(u, v)}^{\sup Y_{(k)}{ }^{u}}=\int_{Y_{(k)^{u}}}\left|D_{k} g(u, v)\right| d \mathcal{L}_{1^{v}}
$$

for $\mathcal{L}_{k-1}$ almost all $u \in Y_{(k)}$,

$$
\int_{\boldsymbol{E}_{k}} N(f, Y, y) d \mathcal{L}_{k} y<\infty .
$$

We infer from Lemma 2.12 that

$$
\int_{Y}\left|D_{k} g(x)\right| d \mathcal{L}_{k} x=\int_{\boldsymbol{B}_{k}} N(f, Y, y) d \mathcal{L}_{k} y<\infty .
$$


This equality may be extended to hold whenever $Y$ is a Borel subset of $E_{k}$ contained in $X$.

Now suppose $W$ is an $\mathcal{L}_{k}$ measurable subset of $X$. Borel subsets $S$ and $T$ of $E_{k}$ may be chosen in such a way that

$$
S \subset W \subset T \subset X, \quad \mathcal{L}_{k}(T-S)=0 .
$$

Since

$$
\begin{aligned}
N(f, S, y) & \leqq N(f, W, y) \leqq N(f, T, y) \quad \text { for } y \in E_{k}, \\
\int_{E_{k}} N(f, S, y) d \mathcal{L}_{k} y & =\int_{S}\left|D_{k} g(x)\right| d \mathcal{L}_{k} x=\int_{W}\left|D_{k} g(x)\right| d \mathcal{L}_{k} x \\
& =\int_{T}\left|D_{k} g(x)\right| d \mathcal{L}_{k} x=\int_{E_{k}} N(f, T, y) d \mathcal{L}_{k} y<\infty,
\end{aligned}
$$

it follows that

$$
\begin{aligned}
& N(f, S, y)=N(f, T, y) \quad \text { for } \mathcal{L}_{k} \text { almost all } y \text { in } E_{k} ; \\
& N(f, W, y) \text { is } \mathcal{L}_{k} \text { measurable in } y, \\
& \int_{W}\left|D_{k} g(x)\right| d \mathcal{L}_{k} x=\int_{E_{k}} N(f, W, y) d \mathcal{L}_{k} y<\infty .
\end{aligned}
$$

PART 5. (ii) implies (i).

2.16 REMARK. A combination of Theorems 2.6, 2.14, and 2.15 reveals that $g$ is $B V T(k)$ on $X$ implies

$$
\int_{X}\left|D_{k} g(x)\right| d \mathcal{L}_{k} x \leqq L(f \mid X)=\int_{E_{k}} S(f, X, y) d \mathcal{L}_{k} y=\int_{E_{k}} N(f, X, y) d \mathcal{L}_{k} y,
$$

equality holding if and only if $g$ is $A C T(k)$.

2.17 Theorem. If $h$ is an $\mathcal{L}_{k}$ measurable function satisfying

$$
-\infty \leqq h(x) \leqq \infty
$$

for $\mathcal{L}_{k}$ almost all $x$ in $E_{k}$, then

$$
g \text { is } A C T(k) \text { on } X \text { implies }
$$

$$
\int_{Y}(h \circ f)(x) \cdot\left|D_{k} g(x)\right| d \mathcal{L}_{k} x=\int_{E_{k}} h(y) \cdot N(f, Y, y) d \mathcal{L}_{k} y
$$

whenever $Y$ is an $\mathcal{L}_{k}$ measurable subset of $X$.

Proof. This statement is an immediate consequence of Theorem 2.15 and [F3, 2.1].

2.18 THEOREM. If for $x \in E_{k}$ and $r>0$ 


$$
C(x, r)=E_{k} \cap\{z|| z-x \mid \leqq r\},
$$

then

$$
\begin{gathered}
g \text { is } B V T(k) \text { on } X \text { implies } \\
\lim _{r \rightarrow 0+} \frac{L[f \mid C(x, r)]}{\alpha(k) r^{k}}=\left|D_{k} g(x)\right| \quad \text { for } \mathcal{L}_{k} \text { almost all } x \in \text { interior } X .
\end{gathered}
$$

Proof. If $U$ is the set of those points $x$ in $X$ for which $D_{k} g(x)$ exists, we know that $U$ is a Borel subset of $E_{k}$ and may define functions $\beta, \gamma$, and $\delta$ as follows:

$$
\begin{aligned}
\beta(Y) & =\int_{E_{k}} N(f, Y, y) d \mathcal{L}_{k} y, \\
\gamma(Y) & =\int_{Y}\left|D_{k} g(x)\right| d \mathcal{L}_{k} x, \\
\delta(Y) & =\int_{E_{k}} N(f, Y-U, y) d \mathcal{L}_{k} y,
\end{aligned}
$$

for $Y$ a Borel subset of $E_{k}$ contained in $X$.

Employing the terminology of Saks [S, p. 30], $\beta, \gamma$, and $\delta$ are additive set functions on the class of Borel subsets of $E_{k}$ contained in $X$,

$$
\gamma \text { is } \mathcal{L}_{k} \text { absolutely continuous, }
$$

and since $\mathcal{L}_{k}(X-U)=0$

$$
\delta \text { is } \mathcal{L}_{k} \text { singular. }
$$

Because of Theorem 2.14

$$
\beta=\gamma+\delta .
$$

Hence using Theorem 2.6 and [S, IV 5.4, 6.3, 7.1] we conclude that

$$
\begin{aligned}
\lim _{\boldsymbol{r} \rightarrow 0+} \frac{L[f \mid C(x, r)]}{\alpha(k) \boldsymbol{r}^{k}} & =\lim _{\boldsymbol{r} \rightarrow 0+} \frac{\beta[C(x, r)]}{\alpha(k) \boldsymbol{r}^{k}} \\
& =\lim _{\boldsymbol{r} \rightarrow 0+} \frac{\gamma[C(x, \boldsymbol{r})]}{\alpha(k) \boldsymbol{r}^{k}}+\lim _{\boldsymbol{r} \rightarrow 0+} \frac{\delta[C(x, \boldsymbol{r})]}{\alpha(k) \boldsymbol{r}^{k}} \\
& =\left|D_{k} g(x)\right|
\end{aligned}
$$

for $\mathcal{L}_{k}$ almost all $x \in$ interior $X$.

2.19 REMARK. If $g$ is BVT, then a computation shows that

$$
J f(x)=\left|D_{k} g(x)\right| \quad \text { for } \mathcal{L}_{k} \text { almost all } x \text { in } X .
$$

In this case the validity of Theorems $2.14,2.15,2.17$, and 2.18 and Remark 
2.16 holds whenever $\left|D_{k} g\right|$ is replaced by $J f$.

2.20 REMARK. If $f \in \Omega_{k}$ were of the form

$$
f(x)=\left(x_{1}, \cdots, x_{j-1}, g(x), x_{j+1}, \cdots, x_{k}\right) \quad \text { for } x \in E_{k},
$$

for $j$ some positive integer less than $k$ and $g$ a continuous real-valued function on $E_{k}$, then the foregoing theory would hold, provided only that the conditions $\operatorname{BVT}(k)$ and $\operatorname{ACT}(k)$ be replaced by $\operatorname{BVT}(j)$ and $\operatorname{ACT}(j)$, and the function $D_{k} g$ be replaced by $D_{j} g$.

3. $k$ dimensional nonparametric surfaces in $E_{k+1}(k>1)$. Each orthogonal projection of $E_{k+1}$ onto $E_{k}$ is of the form

$$
p_{k+1}^{k} \circ R
$$

for some $R \in G_{k+1}$. In fact $p_{\boldsymbol{k}+1}^{k} \circ R$ maps the $k$-dimensional subspace of $E_{k+1}$ spanned by the $k$ tuple of vectors

$$
\left\langle(\operatorname{inv} R)^{1}, \cdots,(\operatorname{inv} R)^{k}\right\rangle
$$

onto $E_{k}$, transforming (inv $\left.R\right)^{i}$ into ${ }^{k} I^{i}$ for $i=1,2, \cdots, k$.

Since for $x \in E_{k+1}$ and $R \in G_{k+1}$,

$$
\left(p_{k+1}^{k} \circ R\right)(x)=\left(x \bullet(\text { inv } R)^{1}, \cdots, x \bullet(\text { inv } R)^{k}\right),
$$

two orthogonal projections $p_{k+1}^{k} \circ S$ and $p_{k+1}^{k} \circ T$ are equal if and only if

$$
(\operatorname{inv} S)^{i}=(\operatorname{inv} T)^{i} \quad \text { for } i=1,2, \cdots, k .
$$

For $m$ a positive integer greater than two, $\Lambda_{m}$ will denote the set of all those elements $R$ of $G_{m}$ for which there exists some $S \in G_{2}$ satisfying

$$
\begin{array}{ll}
R^{i}={ }^{m} I^{i} & \text { for } i=1,2, \cdots, m-2 ; \\
R_{j}^{i}=0 & \text { for } i=m-1, m ; j=1,2, \cdots, m-2 ; \\
R_{j}^{i}=S_{j-m+2}^{i-m+2} & \text { for } i=m-1, m ; j=m-1, m .
\end{array}
$$

If $f$ is a continuous real-valued function on $E_{k}$ and $i$ is a positive integer, then $\bar{f}$ and $\bar{f}_{i}$ will be the functions defined by the relations

$$
\begin{array}{ll}
\bar{f}(x)=\left(x_{1}, x_{2}, \cdots, x_{k}, f(x)\right) & \text { for } x \in E_{k}, \\
\bar{f}_{i}(x)=\left(x_{1}, x_{2}, \cdots, x_{k}, f_{i}(x)\right) & \text { for } x \in E_{k} .
\end{array}
$$

3.1 Sectional assumptions. We let $f$ be a fixed continuous real-valued function on $E_{k}$, and for $j$ a positive integer no greater than $k+1,{ }^{i} R$ will denote that element of $G_{k+1}$ for which

$$
{ }^{i} R(x)=\left(x_{1}, \cdots, x_{j-1}, x_{k+1}, x_{j+1}, \cdots, x_{k}, x_{j}\right) \quad \text { for } x \in E_{k} .
$$

Observe that 
${ }^{k+1} R$ is the identity element of $G_{k+1}$,

$$
p_{k+1}^{k} \circ{ }^{j} R \circ \bar{f} \in \Omega_{k} \quad \text { for } j=1,2, \cdots, k+1 .
$$

3.2 Lемма. If $Y$ is a bounded open convex subset of $E_{k}$ and $i$ is a positive integer, then

$$
S \in \Lambda_{k+1} \text { implies }
$$

(i) $\int_{E_{k}} N\left(p_{k+1}^{k} \circ S \circ \bar{f}_{i}, \quad Y, x\right) d \mathcal{L}_{k} x$

$$
\leqq \alpha(k)^{-1} i^{k} \int_{K_{i}} \int_{E_{k}} N\left(p_{k+1}^{k} \circ S \circ \bar{f}, Y_{z}, x\right) d \mathcal{L}_{k} x d \mathcal{L}_{k} z,
$$

(ii) $N\left(p_{k+1}^{k} \circ S \circ \bar{f}, Y, x\right)=S\left(p_{k+1}^{k} \circ S \circ \bar{f}, Y, x\right)$ for $\mathcal{L}_{k}$ almost all $x$ in $E_{k}$.

Proof. We know that

$$
\begin{aligned}
\left(p_{k+1}^{k} \circ S\right)(w) & =\left(w \bullet(\operatorname{inv} S)^{1}, \cdots, w \bullet(\operatorname{inv} S)^{k}\right) \\
& =\left(w_{1}, w_{2}, \cdots, w_{k-1}, w_{k} \cdot S_{k}^{k}+w_{k+1} \cdot S_{k}^{k+1}\right)
\end{aligned}
$$

for $w \in E_{k+1}$. Thus

$$
\begin{aligned}
\left(p_{k+1}^{k} \circ S \circ \bar{f}\right)(x) & =\left(p_{k+1}^{k} \circ S\right)\left(x_{1}, x_{2}, \cdots, x_{k}, f(x)\right) \\
& =\left(x_{1}, x_{2}, \cdots, x_{k-1}, S_{k}^{k} \cdot x_{k}+S_{k}^{k+1} \cdot f(x)\right)
\end{aligned}
$$

whenever $x \in E_{k}$.

We may infer then that $p_{k+1}^{k} \circ S \circ \bar{f} \in \Omega_{k}$. Moreover upon letting $F(x)$ $=S_{k}^{k} \cdot x_{k}+S_{k}^{k+1} \cdot f(x)$ for $x \in E_{k}$, a check reveals that

$$
\begin{array}{rlr}
F_{i}(x) & =S_{k}^{k} \cdot x_{k}+S_{k}^{k+1} \cdot f_{i}(x) & \text { for } x \in E_{k}, \\
\cdot\left(p_{k+1}^{k} \circ S \circ \bar{f}\right)_{i} & =p_{k+1}^{k} \circ S \circ \bar{f}_{i},
\end{array}
$$

for every positive integer $i$. Accordingly statements (i) and (ii) follow from Lemma 2.2 and Theorem 2.4.

3.3 Lemma. If $R \in G_{k+1}$, then there exist functions $S, T$ and $U$ for which

$$
\begin{gathered}
S \in \Lambda_{k+1}, \quad T \in G_{k+1}, \quad U \in G_{k}, \\
T\left({ }^{k+1} I^{k+1}\right)={ }^{k+1} I^{k+1}, \\
p_{k+1}^{k} \circ R=U \circ p_{k+1}^{k} \circ S \circ T .
\end{gathered}
$$

Proof. We know for any two vector subspaces of a vector space that the sum of their dimensions is equal to the sum of the dimensions of the vector spaces generated respectively by their union and intersection.

Accordingly if $Z$ is the subspace of $E_{k+1}$ spanned by the $k$-tuple of vectors

$$
\left\langle{ }^{k+1} I^{1},{ }^{k+1} I^{2}, \cdots,{ }^{k+1} I^{k}\right\rangle
$$

and $K$ the subspace of $E_{k+1}$ spanned by the $k$-tuple of vectors 


$$
\left\langle(\operatorname{inv} R)^{1},(\operatorname{inv} R)^{2}, \cdots,(\operatorname{inv} R)^{k}\right\rangle,
$$

then the dimension of the subspace $K \cap Z$ equals either $k$ or $k-1$.

If dimension $(K \cap Z)=k$, then $K$ coincides with $Z$, and we may select $T \in G_{k+1}$ so that $T$ is the rotation of $Z$ which transforms (inv $R$ ) $^{i}$ into ${ }^{k+1} I^{i}$ for $i=1,2, \cdots, k$. Letting $U$ denote the identity map of $E_{k}$ and $S$ the identity map of $E_{k+1}$ we may easily check that

$$
\begin{gathered}
p_{k+1}^{k} \circ R=U \circ\left(p_{k+1}^{k} \circ R \circ \operatorname{inv} T\right) \circ T, \\
p_{k+1}^{k} \circ R \circ \operatorname{inv} T=p_{k+1}^{k} \circ S .
\end{gathered}
$$

Now suppose that the dimension of $K \cap Z$ is $k-1$.

Using the transitivity of the orthogonal group, we can choose functions $A, U$, and $T$ for which

$$
A \in G_{k+1}, \quad A^{*}(K)=K,
$$

$\left\langle(A \circ \operatorname{inv} R)^{1},(A \circ \operatorname{inv} R)^{2}, \cdots,(A \circ \operatorname{inv} R)^{k-1}\right\rangle$ spans $K \cap Z$,

$$
U \in G_{k} \text {, }
$$

$$
U\left[\left(p_{k+1}^{k} \circ R\right)\left((\operatorname{inv} A \circ \operatorname{inv} R)^{i}\right)\right]={ }^{k} I^{i} \quad \text { for } i=1,2, \cdots, k \text {, }
$$

$$
\begin{aligned}
& T \in G_{k+1}, \quad T^{*}(Z)=Z, \\
& T\left[(A \circ \operatorname{inv} R)^{i}\right]={ }^{k+1} I^{i} \quad \text { for } i=1,2, \cdots, k-1 .
\end{aligned}
$$

Since $(T \circ A \circ \text { inv } R)^{k}$ lies in the orthogonal complement of the space generated by $\left\langle{ }^{k+1} I^{1},{ }^{k+1} I^{2}, \cdots,{ }^{k+1} I^{k-1}\right\rangle$, an element $B$ of $G_{2}$ may be picked such that

$$
(T \circ A \circ \operatorname{inv} R)_{k-1+i}^{k}=B_{i}^{1} \quad \text { for } i=1,2 .
$$

Then defining $S$ to be the element of $G_{k+1}$ satisfying

$$
\begin{array}{ll}
(\text { inv } S)^{i}={ }^{k+1} I^{i} & \text { for } i=1,2, \cdots, k-1 ; \\
(\text { inv } S)_{j}^{i}=0 & \text { for } i=k, k+1 ; j=1,2, \cdots, k-1 ; \\
(\text { inv } S)_{j}^{i}=B_{j-k+1}^{i-k+1} & \text { for } i=k, k+1 ; j=k, k+1 ;
\end{array}
$$

we may infer that $S$ (along with inv $S$ ) is an element of $\Lambda_{k+1}$.

Inasmuch as

$$
\begin{aligned}
(\operatorname{inv}(S \circ T))^{i} & =(\operatorname{inv} T \circ \operatorname{inv} S)^{i} \\
& =\operatorname{inv} T\left[(\operatorname{inv} S)^{i}\right] \\
& =\operatorname{inv} T\left[(T \circ A \circ \operatorname{inv} R)^{i}\right] \\
& =(A \circ \operatorname{inv} R)^{i} \\
& =(\operatorname{inv}(R \circ \operatorname{inv} A))^{i} \quad \text { for } i=1,2, \cdots, k,
\end{aligned}
$$


it follows that

$$
p_{k+1}^{k} \circ S \circ T=\stackrel{k}{k} \circ R \circ \operatorname{inv} A \text {. }
$$

Let $i$ be a positive integer no greater than $k$. To complete the proof it is sufficient to show the equality of the functions $p_{k+1}^{k} \circ R$ and $U \circ p_{k+1}^{k} \circ S \circ T$ on the vector

$$
(\operatorname{inv} R)^{i}
$$

We compute:

$$
\begin{aligned}
\left(U \circ p_{k+1}^{k} \circ S \circ T\right)\left[(\operatorname{inv} R)^{i}\right] & =\left(U \circ p_{k+1}^{k} \circ R \circ \operatorname{inv} A\right)\left[(\operatorname{inv} R)^{i}\right] \\
& =U\left[\left(p_{k+1}^{k} \circ R\right)\left((\operatorname{inv} A \circ \operatorname{inv} R)^{i}\right)\right] \\
& ={ }^{k} I^{i} .
\end{aligned}
$$

3.4 Lemma. If $Y$ is a bounded open convex subset of $E_{k}$ and $i$ is a positive integer, then

$$
R \in G_{k+1} \text { implies }
$$

$$
\begin{aligned}
\int_{E_{k}} N\left(p_{k+1}^{k} \circ R \circ \bar{f}_{i}, Y, x\right) & d \mathcal{L}_{k} x \\
& \leqq \alpha(k)^{-1} i^{k} \int_{K_{i}} \int_{E_{k}} N\left(p_{k+1}^{k} \circ S \circ \bar{f}, Y_{z}, x\right) d \mathcal{L}_{k} x d \mathcal{L}_{k} z .
\end{aligned}
$$

Proof. Suppose $R \in G_{k+1}$. We use the preceding lemma to select functions $U, S, T, t$ such that

$$
\begin{gathered}
U \in G_{k}, \quad S \in \Lambda_{k+1}, \quad T \in G_{k+1}, \quad t \in G_{k}, \\
T\left({ }^{k+1} I^{k+1}\right)={ }^{k+1} I^{k+1},
\end{gathered}
$$

if $w \in E_{k}, z \in E_{k}$, and $v \in E_{1}$, then $t(z)=w$ if and only if $T\left(z_{1}, z_{2}, \cdots, z_{k}, v\right)$ $=\left(w_{1}, w_{2}, \cdots, w_{k}, v\right)$,

$$
p_{k+1}^{k} \circ R=U \circ p_{k+1}^{k} \circ S \circ \operatorname{inv} T .
$$

We shall denote

$$
\operatorname{inv} t(z)=z^{\prime} \quad \text { whenever } z \in E_{k},
$$

and for $i$ a positive integer, we define the functions $F, F_{i}, \bar{F}, \bar{F}_{i}$ by the formulae

$$
\begin{aligned}
F & =f \circ t, \quad F_{i}=f_{i} \circ t, & & \\
\bar{F}(x) & =\left(x_{1}, x_{2}, \cdots, x_{k}, F(x)\right) & & \text { for } x \in E_{k}, \\
\bar{F}_{i}(x) & =\left(x_{1}, x_{2}, \cdots, x_{k}, F_{i}(x)\right) & & \text { for } x \in E_{k} .
\end{aligned}
$$

The rest of the proof is divided into three parts. 
PART 1. If $V \in G_{k}, B \subset E_{k}$, and $z \in E_{k}$, then

$$
\left[(\operatorname{inv} V)^{*}(B)\right]_{z}=(\operatorname{inv} V)^{*}\left[B_{V(z)}\right] \text {. }
$$

Proof.

$$
\begin{aligned}
(\operatorname{inv} V)^{*}\left[B_{V(z)}\right] & =(\operatorname{inv} V)^{*}(\{y \mid y-V(z) \in B\}) \\
& =\{x \mid V(x)-V(z) \in B\} \\
& =\left\{x \mid x-z \in(\operatorname{inv} V)^{*}(B)\right\} \\
& =\left[(\operatorname{inv} V)^{*}(B)\right]_{z .}
\end{aligned}
$$

PART 2. (i) $p_{k+1}^{k} \circ R \circ \bar{f}=U \circ p_{k+1}^{k} \circ S \circ \bar{F} \circ$ inv $t$,

(ii) $p_{k+1}^{k} \circ R \circ \bar{f}_{i}=U \circ p_{k+1}^{k} \circ S \circ \bar{F}_{i} \circ$ inv $t$.

Proof. It is sufficient to verify (ii). Let $z \in E_{k}$, then

$$
\begin{aligned}
\left(p_{k+1}^{k} \circ R \circ \bar{f}_{i}\right)(z) & =\left(p_{k+1}^{k} \circ R\right)\left(z_{1}, z_{2}, \cdots, z_{k}, f_{i}(z)\right) \\
& =\left(U \circ p_{k+1}^{k} \circ S \circ \operatorname{inv} T\right)\left(z_{1}, z_{2}, \cdots, z_{k}, f_{i}(z)\right) \\
& =\left(U \circ p_{k+1}^{k} \circ S\right)\left(z_{1}^{\prime}, z_{2}^{\prime}, \cdots, z_{k}^{\prime}, f_{i}(z)\right) \\
& =\left(U \circ p_{k+1}^{k} \circ S\right)\left(z_{1}^{\prime}, z_{2}^{\prime}, \cdots, z_{k}^{\prime}, F_{i}\left(z^{\prime}\right)\right) \\
& =\left(U \circ p_{k+1}^{k} \circ S \circ \bar{F}_{i}\right)\left(z^{\prime}\right) \\
& =\left(U \circ p_{k+1}^{k} \circ S \circ \bar{F}_{i} \circ \operatorname{inv} t\right)(z) .
\end{aligned}
$$

PART 3.

$$
\begin{aligned}
\int_{E_{k}} N\left(p_{k+1}^{k} \circ R \circ \bar{f}_{i}, Y, x\right) & d \mathcal{L}_{k} x \\
& \leqq \alpha(k)^{-1} i^{k} \int_{K_{i}} \int_{E_{k}} N\left(p_{k+1}^{k} \circ S \circ \bar{f}, Y_{z}, x\right) d \mathcal{L}_{k} x d \mathcal{L}_{k} z .
\end{aligned}
$$

Proof. With the aid successively of Part 2, Lemma 3.2 applied to $\bar{F}_{i}$, Part 1 , the fact that

$$
J t(z)=1
$$

whenever $z \in E_{k}$,

and Part 2 again, we check that

$$
\begin{aligned}
\int_{E_{k}} N\left(p_{k+1}^{k} \circ R\right. & \left.\circ \bar{f}_{i}, Y, x\right) d \mathcal{L}_{k} x \\
& =\int_{E_{k}} N\left(U \circ p_{k+1}^{k} \circ S \circ \bar{F}_{i} \circ \operatorname{inv} t, Y, x\right) d \mathcal{L}_{k} x \\
& =\int_{E_{k}} N\left(p_{k+1}^{k} \circ S \circ \bar{F}_{i} \circ \operatorname{inv} t, Y, x\right) d \mathcal{L}_{k} x
\end{aligned}
$$




$$
\begin{aligned}
& =\int_{E_{k}} N\left(p_{k+1}^{k} \circ S \circ \bar{F}_{i},(\operatorname{inv} t)^{*}(Y), x\right) d \mathcal{L}_{k} x \\
& \leqq \alpha(k)^{-1} i^{k} \int_{K_{i}} \int_{E_{k}} N\left(p_{k+1}^{k} \circ S \circ \bar{F},\left[(\operatorname{inv} t)^{*}(Y)\right]_{z}, x\right) d \mathcal{L}_{k} x d \mathcal{L}_{k} z \\
& =\alpha(k)^{-1} i^{k} \int_{K_{i}} \int_{E_{k}} N\left(p_{k+1}^{k} \circ S \circ \bar{F},(\operatorname{inv} t)^{*}\left[Y_{t(z)}\right], x\right) d \mathcal{L}_{k} x d \mathcal{L}_{k} z \\
& =\alpha(k)^{-1} i^{k} \int_{K_{i}} \int_{E_{k}} N\left(p_{k+1}^{k} \circ S \circ \bar{F} \circ \operatorname{inv} t, Y_{t(z)}, x\right) d \mathcal{L}_{k} x d \mathcal{L}_{k} z \\
& =\alpha(k)^{-1} i^{k} \int_{K_{i}} J t(z) \int_{E_{k}} N\left(p_{k+1}^{k} \circ S \circ \bar{F} \circ \operatorname{inv} t, Y_{t(z)}, x\right) d \mathcal{L}_{k} x d \mathcal{L}_{k} z \\
& =\alpha(k)^{-1} i^{k} \int_{i^{*}\left(K_{i}\right)} \int_{E_{k}} N\left(p_{k+1}^{k} \circ S \circ \bar{F} \circ \operatorname{inv} t, Y_{z}, x\right) d \mathcal{L}_{k} x d \mathcal{L}_{k} z \\
& =\alpha(k)^{-1} i^{k} \int_{K_{i}} \int_{E_{E_{k}}} N\left(U \circ p_{k+1}^{k} \circ S \circ \bar{F} \circ \operatorname{inv} t, Y_{z}, x\right) d \mathcal{L}_{k} x d \mathcal{L}_{k} z \\
& =\alpha(k)^{-1} i^{k} \int_{K_{i}} \int_{E_{k}} N\left(p_{k+1}^{k} \circ R \circ \bar{f}, Y, x\right) d \mathcal{L}_{k} x d \mathcal{L}_{k} z .
\end{aligned}
$$

3.5 Theorem. If $Y$ is a bounded open convex subset of $E_{k}$, then $R \in G_{k+1}$ implies $N\left(p_{k+1}^{k} \circ R \circ \bar{f}, Y, x\right)=S\left(p_{k+1}^{k} \circ R \circ \bar{f}, Y, x\right)$ for $\mathcal{L}_{k}$ almost all $x \in E_{k}$.

Proof. Let $R \in G_{k+1}$. Just as in the preceding lemma we may select functions $U, S, t$ and define functions $F$ and $\bar{F}$ so that

$$
\begin{gathered}
S \in \Lambda_{k+1}, \quad U \in G_{k}, \quad t \in G_{k}, \\
F=f \circ t, \\
p_{k+1}^{k} \circ R \circ \bar{f}=U \circ p_{k+1}^{k} \circ S \circ \bar{F} \circ \operatorname{inv} t .
\end{gathered}
$$

Then with the help of Lemma 3.2 we find that

$$
\begin{aligned}
N\left(p_{k+1}^{k} \circ R\right. & \circ \bar{f}, Y, x) \\
& =N\left(U \circ p_{k+1}^{k} \circ S \circ \bar{F} \circ \operatorname{inv} t, Y, x\right) \\
& =N\left(p_{k+1}^{k} \circ S \circ \bar{F},(\operatorname{inv} t)^{*}(Y), x\right) \\
& =S\left(p_{k+1}^{k} \circ S \circ \bar{F},(\operatorname{inv} t)^{*}(Y), x\right) \\
& =S\left(U \circ p_{k+1}^{k} \circ S \circ \bar{F} \circ \operatorname{inv} t, Y, x\right) \\
& =S\left(p_{k+1}^{k} \circ R \circ \bar{f}, Y, x\right)
\end{aligned}
$$$$
\text { for } \mathcal{L}_{k} \text { almost all } x \text { in } E_{k}
$$$$
\text { for } \mathcal{L}_{k} \text { almost all } x \text { in } E_{k}
$$$$
\text { for } \mathcal{L}_{k} \text { almost all } x \text { in } E_{k} \text {. }
$$

3.6 REMARK. If $Y \subset E_{k}$ is the interior of $a k$ cell and $R \in G_{k+1}$, then 


$$
\begin{aligned}
\int_{E_{k}} N\left(p_{k+1}^{k} \circ R \circ \bar{f}, Y, x\right) d \mathcal{L}_{k} x & =\int_{E_{k}} S\left(p_{k+1}^{k} \circ R \circ \bar{f}, Y, x\right) d \mathcal{L}_{k} x \\
& =L\left(p_{k+1}^{k} \circ R \circ \bar{f} \mid Y\right) .
\end{aligned}
$$

For if $S, U, t$, and $F$ are chosen as in Theorem 3.5, it then follows by Theorems 2.6 and 3.5 and certain invariance properties of the Lebesgue area that

$$
\begin{aligned}
L\left(p_{k+1}^{k} \circ R \circ \bar{f} \mid Y\right) & =L\left(U \circ p_{k+1}^{k} \circ S \circ \bar{F} \circ \operatorname{inv} t \mid Y\right)=L\left(p_{k+1}^{k} \circ S \circ \bar{F} \circ \operatorname{inv} t \mid Y\right) \\
& =L\left(p_{k+1}^{k} \circ S \circ \bar{F} \mid(\operatorname{inv} t)^{*}(Y)\right) \\
& =\int_{E_{k}} N\left(p_{k+1}^{k} \circ S \circ \bar{F},(\operatorname{inv} t)^{*}(Y), x\right) d \mathcal{L}_{k} x \\
& =\int_{E_{k}} N\left(U \circ p_{k+1}^{k} \circ S \circ \bar{F} \circ \operatorname{inv} t, Y, x\right) d \mathcal{L}_{k} x \\
& =\int_{E_{k}} N\left(p_{k+1}^{k} \circ R \circ \bar{f}, Y, x\right) d \mathcal{L}_{k} x \\
& =\int_{E_{k}} S\left(p_{k+1}^{k} \circ R \circ \bar{f}, Y, x\right) d \mathcal{L}_{k} x .
\end{aligned}
$$

3.7 Lemma. If $X \subset E_{k}$ is a $k$ cell with boundary $\dot{X}$, then

$$
\mathfrak{H}_{k+1}^{k}\left[\bar{f}^{*}(\dot{X})\right]=0 .
$$

Proof. The method of proof selected for Lemma 2.5 included an explicit proof of this statement.

3.8 THEOREM. If $X \subset E_{k}$ is a $k$ cell, then

$$
\begin{aligned}
\beta(k+1, k)^{-1} \int_{a_{k+1}} & \int_{E_{k}} N\left(p_{k+1}^{k} \circ R \circ \bar{f}, X, x\right) d \mathcal{L}_{k} x d \phi_{k+1} R \\
& =\beta(k+1, k)^{-1} \int_{a_{k+1}} \int_{E_{k}} S\left(p_{k+1}^{k} \circ R \circ \bar{f}, X, x\right) d \mathcal{L}_{k} x d \phi_{k+1} R \\
& =L(\bar{f} \mid X) \\
& =\lim _{\rightarrow \rightarrow \infty} L\left(\bar{f}_{j} \mid X\right) .
\end{aligned}
$$

Proof. Let $Y=$ interior $X$. Then from Lemma 3.5 we know that

$$
\int_{E_{k}} N\left(p_{k+1}^{k} \circ R \circ \bar{f}, Y, x\right) d \mathcal{L}_{k} x=\int_{E_{k}} S\left(p_{k+1}^{k} \circ R \circ \bar{f}, Y, x\right) d \mathcal{L}_{k} x
$$


for $R \in G_{k+1}$. Therefore

$$
\begin{aligned}
\beta(k+1, k)^{-1} \int_{G_{k+1}} & \int_{E_{k}} N\left(p_{k+1}^{k} \circ R \circ \bar{f}, Y, x\right) d \mathcal{L}_{k} x d \phi_{k+1} R \\
= & \beta(k+1, k)^{-1} \int_{G_{k+1}} \int_{E_{k}} S\left(p_{k+1}^{k} \circ R \circ \bar{f}, Y, x\right) d \mathcal{L}_{k} x d \phi_{k+1} R .
\end{aligned}
$$

We suppose that $A$ is an open interval of $E_{k}$ for which

$$
\text { closure } A \subset Y \text {, }
$$

and select a positive integer $i$ so large that

$$
A_{z} \subset Y \quad \text { whenever } z \in K_{i} \text {. }
$$

Then with the help of $[F 4,6.13],[F 4,4.5]$, and Lemma 3.4 we obtain

$$
\begin{aligned}
& L\left(\bar{f}_{i} \mid \text { closure } A\right)=\int_{A} J \bar{f}_{i}(x) d \mathcal{L}_{k} x \\
& \quad=\beta(k+1, k)^{-1} \int_{G_{k+1}} \int_{E_{k}} N\left(p_{k+1}^{k} \circ R \circ \bar{f}_{i}, A, x\right) d \mathcal{L}_{k} x d \phi_{k+1} R \\
& \quad \leqq \beta(k+1, k)^{-1} \int_{G_{k+1}} \alpha(k)^{-1} i^{k} \int_{K_{i}} \int_{E_{k}} N\left(p_{k+1}^{k} \circ R \circ \bar{f}, A_{z}, x\right) d \mathcal{L}_{k} x d \mathcal{L}_{k} z d \phi_{k+1} R \\
& \quad \leqq \beta(k+1, k)^{-1} \int_{G_{k+1}} \int_{E_{k}} N\left(p_{k+1}^{k} \circ R \circ \bar{f}, Y, x\right) d \mathcal{L}_{k} x d \phi_{k+1} R .
\end{aligned}
$$

From the lower semi-continuity of $L$ on $C_{k}(X)$ it follows that $L(\bar{f} \mid$ closure $A) \leqq \liminf _{i \rightarrow \infty} L\left(\bar{f}_{i} \mid\right.$ closure $\left.A\right)$

$$
\leqq \beta(k+1, k)^{-1} \int_{G_{k+1}} \int_{\boldsymbol{E}_{k}} N\left(p_{k+1}^{k} \circ R \circ \bar{f}, Y, x\right) d \mathcal{L}_{k} x d \phi_{k+1} R .
$$

As a consequence of the arbitrary nature of $A$

$$
\begin{aligned}
L(\bar{f} \mid Y) & \leqq \beta(k+1, k)^{-1} \int_{G_{k+1}} \int_{E_{k}} N\left(p_{k+1}^{k} \circ R \circ \bar{f}, Y, x\right) d \mathcal{L}_{k} x d \phi_{k+1} R \\
& =\beta(k+1, k)^{-1} \int_{G_{k+1}} \int_{E_{k}} S\left(p_{k+1}^{k} \circ R \circ \bar{f}, Y, x\right) d \mathcal{L}_{k} x d \phi_{k+1} R \\
& \leqq L(\bar{f} \mid Y) .
\end{aligned}
$$

Because of Lemma 3.7 this last relationship holds when $Y$ is replaced by $X$.

Using the foregoing results the other part of the statement may be proved exactly as the corresponding statement was proved in Theorem 2.6. 
3.9 REmark. For $X \subset E_{k}$ a $k$ cell, the results of the last theorem allow us, using the notation of $[\mathrm{F} 6]$, to state $L(\bar{f} \mid X)=M^{* *}(\bar{f} \mid X)=S^{* *}(\bar{f} \mid X)=U^{* *}(\bar{f} \mid X)=V^{* *}(\bar{f} \mid X)=N^{* *}(\bar{f} \mid X)$.

3.10 REMARK. If $Y$ is an analytic subset of $E_{k}$, then

$$
\mathcal{f}_{k+1}^{k}\left[\bar{f}^{*}(Y)\right]=\beta(k+1, k)^{-1} \int_{G_{k+1}} \int_{E_{k}} N\left(p_{k+1}^{k} \circ R \circ \bar{f}, Y, x\right) d \mathcal{L}_{k} x d \phi_{k+1} R .
$$

Since $\bar{f}^{*}(Y)$ is an analytic subset of $E_{k+1}$, the univalence of $\bar{f}$ implies

$$
\begin{aligned}
\mathcal{F}_{k+1}^{k}\left[\bar{f}^{*}(Y)\right] & =\beta(k+1, k)^{-1} \int_{G_{k+1}} \int_{E_{k}} N\left(p_{k+1}^{k} \circ R, \bar{f}^{*}(Y), x\right) d \mathcal{L}_{k} x d \phi_{k+1} R \\
& =\beta(k+1, k)^{-1} \int_{G_{k+1}} \int_{E_{k}} N\left(p_{k+1}^{k} \circ R \circ \bar{f}, Y, x\right) d \mathcal{L}_{k} x d \phi_{k+1} R .
\end{aligned}
$$

3.11 Theorem. If $X \subset E_{k}$ is a $k$ cell and $A$ is a Borel subset of $E_{k+1}$, then

$$
\mathcal{f}_{k+1}^{k}\left[A \cap \bar{f}^{*}(X)\right]=\Gamma_{k+1}^{k}\left[A \cap \bar{f}^{*}(X)\right] \text {. }
$$

Proof. Since in general $\mathcal{F}_{k+1}^{k}$ is dominated by $\Gamma_{k+1}^{k}$, it is sufficient to assume

$$
\mathcal{F}_{k+1}^{k}\left[A \cap \bar{f}^{*}(X)\right]<\infty,
$$

and to show that

$$
\mathcal{F}_{k+1}^{k}\left[A \cap \bar{f}^{*}(X)\right] \geqq \Gamma_{k+1}^{k}\left[A \cap \bar{f}^{*}(X)\right] .
$$

We divide the proof of this into three parts.

PART 1. If $R \in G_{k+1}$, then

$$
L(\bar{f} \mid X) \geqq \int_{E_{k}} S\left(p_{k+1}^{k} \circ R \circ \bar{f}, X, x\right) d \mathcal{L}_{k} x .
$$

Proof. Let $R \in G_{k+1}$. Select a sequence

$$
P_{1}, P_{2}, \cdots \text {, }
$$

of polyhedra of $C_{k}(X)$ for which

$$
\lim _{i \rightarrow \infty} P_{i}=\bar{f} \mid X, \quad \liminf _{i \rightarrow \infty} L\left(P_{i}\right)=L(\bar{f} \mid X) .
$$

For each positive integer $i, p_{k+1}^{k} \circ R \circ P_{i}$ is a polyhedron and

$$
\lim _{i \rightarrow \infty}\left(p_{k+1}^{k} \circ R \circ P_{i}\right)=p_{k+1}^{k} \circ R \circ \bar{f} \mid X .
$$

Suppose $\cup_{j=1}^{m_{i}} T_{j}$ is the simplicial triangulation of $X$ associated with $P_{i}$. 
From the Lipschitzian character of $p_{k+1}^{k} \circ R$ we may infer that

$$
\begin{aligned}
L(\bar{f} \mid X) & =\liminf _{i \rightarrow \infty} \sum_{j=1}^{m_{i}} \mathcal{F}_{k+1}^{k}\left[P_{i}^{*}\left(T_{j}\right)\right] \\
& \geqq \liminf _{i \rightarrow \infty} \sum_{j=1}^{m_{i}} \mathcal{F C}_{k+1}^{k}\left[\left(p_{k+1}^{k} \circ R \circ P_{i}\right)^{*}\left(T_{j}\right)\right] \\
& \geqq L\left(p_{k+1}^{k} \circ R \circ \bar{f} \mid X\right) .
\end{aligned}
$$

We know that the $k$-dimensional Lebesgue area and the $k$-dimensional stable area are lower semi-continuous functions on $C_{k}(X)$, and that both are extensions of the classical area integral over the class of polyhedra. The Lebesgue area being numerically the largest of all such lower semi-continuous extensions, we may conclude that

$$
L\left(p_{k+1}^{k} \circ R \circ \bar{f} \mid X\right) \geqq \int_{E_{k}} S\left(p_{k+1}^{k} \circ R \circ \bar{f}, X, x\right) d \mathcal{L}_{k} x .
$$

PART 2. If $B$ is a Borel subset of $E_{k}$ and $R \in G_{k+1}$, then

$$
\mathcal{f}_{k+1}^{k}\left[\bar{f}^{*}(B)\right] \geqq \int_{E_{k}} N\left(p_{k+1}^{k} \circ R \circ \bar{f}, B, x\right) d \mathcal{L}_{, k} x .
$$

Proof. Using Remark 3.10, Theorem 3.8, and Part 1,

$$
\begin{aligned}
\mathcal{F}_{k+1}^{k}\left[\bar{f}^{*}(Y)\right] & =L(\bar{f} \mid Y) \geqq \int_{E_{k}} S\left(p_{k+1}^{k} \circ R \circ \bar{f}, Y, x\right) d \mathcal{L}_{k} x \\
& =\int_{E_{k}} N\left(p_{k+1}^{k} \circ R \circ \bar{f}, Y, x\right) d \mathcal{L}_{k} x
\end{aligned}
$$

whenever $Y \subset E_{k}$ is a $k$ cell.

The inequality between the first and last members of this string may be extended (by the customary methods) to hold whenever $Y$ is a Borel subset of $E_{k}$.

PART 3. $\mathcal{F}_{k+1}^{k}\left[A \cap \bar{f}^{*}(X)\right] \geqq \Gamma_{k+1}^{k}\left[A \cap \bar{f}^{*}(X)\right]$.

Proof. Suppose $B$ is a Borel subset of $E_{k+1}$ and $B^{\prime}=($ inv $\bar{f}) *\left[B \cap \bar{f}^{*}(X)\right]$. Then using Part 2

$$
\begin{array}{rlrl}
\mathcal{F}_{k+1}^{k}\left[B \cap \bar{f}^{*}(X)\right] & =\mathcal{F}_{k+1}^{k}\left[\bar{f}^{*}\left(B^{\prime}\right)\right] \geqq \int_{E_{k}} N\left(p_{k+1}^{k} \circ R \circ \bar{f}, B^{\prime}, x\right) d \mathcal{L}_{k} x \\
& \geqq \mathcal{L}_{k}\left[\left(p_{k+1}^{k} \circ R \circ \bar{f}\right)^{*}\left(B^{\prime}\right)\right] \\
& =\mathcal{L}_{k}\left[\left(p_{k+1}^{k} \circ R\right)^{*}\left(B \cap \bar{f}^{*}(X)\right)\right] & \\
\mathcal{F}_{k+1}^{k}\left[B \cap \bar{f}^{*}(X)\right] & \geqq \gamma_{k+1}^{k}\left[B \cap \bar{f}^{*}(X)\right] . & \text { for } R \in G_{k+1},
\end{array}
$$

It follows that 


$$
\mathcal{f}_{k+1}^{k}\left[A \cap \bar{f}^{*}(X)\right] \geqq \Gamma_{k+1}^{k}\left[A \cap \bar{f}^{*}(X)\right] \text {. }
$$

3.12 Lemma. If $B$ is a Borel subset of $E_{k}$ and $R \in G_{k+1}$, then

$$
\begin{aligned}
\int_{E_{k}} N\left(p_{k+1}^{k} \circ R \circ \bar{f}, B, y\right) d \mathcal{L}_{k} y & \leqq \mathcal{F}_{k+1}^{k}\left[\bar{f}^{*}(B)\right] \\
& \leqq \sum_{i=1}^{k+1} \int_{E_{k}} N\left(p_{k+1}^{k} \circ{ }^{i} R \circ \bar{f}, B, y\right) d \mathcal{L}_{k} y .
\end{aligned}
$$

Proof. The first inequality is a restatement of Part 2 of the preceding theorem.

Let $Y \subset E_{k}$ be a $k$ cell with boundary $\dot{Y}$. With the help of $[\mathrm{F} 4,6.13]$ we infer that

$$
\begin{aligned}
L\left(\bar{f}_{i} \mid Y\right) & =\int_{Y-\dot{Y}} J \bar{f}_{j}(x) d \mathcal{L}_{k} x \\
& =\int_{Y-\dot{Y}}\left[\sum_{i=1}^{k+1}\left\{J\left(p_{k+1}^{k} \circ \circ^{i} R \circ \bar{f}_{j}\right)(x)\right\}^{2}\right]^{1 / 2} d \mathcal{L}_{k} x \\
& \leqq \int_{Y-\dot{Y}} \sum_{i=1}^{k+1} J\left(p_{k+1}^{k} \circ{ }^{i} R \circ \bar{f}_{j}\right)(x) d \mathcal{L}_{k} x \\
& =\sum_{i=1}^{k+1} L\left(p_{k+1}^{k} \circ^{i} R \circ \bar{f}_{j} \mid Y\right)
\end{aligned}
$$

whenever $j$ is a positive integer. Letting $j \rightarrow \infty$, it follows from Theorems 3.10 and 2.8 that $L(\bar{f} \mid Y) \leqq \sum_{i=1}^{k+1} L\left(p_{k+1}^{k} \circ{ }^{i} R \circ \bar{f} \mid Y\right)$. We see by reference to Theorems 2.6, 3.8, and Remark 3.10 that

$$
\mathcal{F}_{k+1}^{k}\left[\bar{f}^{*}(Y)\right] \leqq \sum_{i=1}^{k+1} \int_{Y} N\left(p_{k+1}^{k} \circ{ }^{i} R \circ \bar{f}, Y, y\right) d \mathcal{L}_{k} y .
$$

The proof is completed by extending this inequality to hold whenever $Y$ is a Borel subset of $E_{k}$.

3.13 Sectional assumption. For the rest of this section $X \subset E_{k}$ will denote a $k$ cell.

3.14 Lemma. If $j$ is a positive integer not exceeding $k$, then

$$
\begin{aligned}
& \int_{X_{(i)}} T_{\left.v=\sin f(j)^{2}\right)^{u}}^{\sup X_{(j)}} f\left(u_{1}, \cdots, u_{j-1}, v, u_{j+1}, \cdots, u_{k}\right) d \mathcal{L}_{k-1} u
\end{aligned}
$$

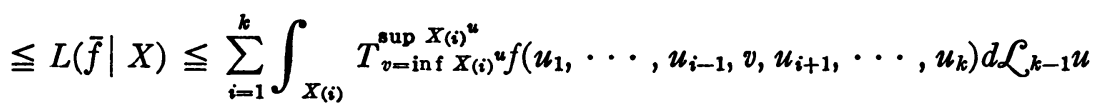

$$
\begin{aligned}
& +\mathcal{L}_{k}(X) \text {. }
\end{aligned}
$$


Proof. We recall for $i=1,2, \cdots, k$, that

$$
\begin{gathered}
\left(p_{k+1}^{k} \circ{ }^{i} R \circ \bar{f}\right)(x)=\left(x_{1}, \cdots, x_{i-1}, f(x), x_{i+1}, \cdots, x_{k}\right) \\
p_{k+1}^{k} \circ{ }^{i} R \circ \bar{f} \in \Omega_{k} .
\end{gathered} \quad \text { for } x \in E_{k},
$$

Consequently we may use Part 2 of Lemma 2.2, Theorem 3.8, and the preceding lemma to compute:

$$
\begin{aligned}
\int_{X_{(i)}} & T_{v=\inf X_{(i)}}^{\sup X_{(j)}^{u}} f\left(u_{1}, \cdots, u_{j-1}, v, u_{j+1}, \cdots, u_{k}\right) d \mathcal{L}_{k-1} u \\
& =\int_{E_{k}} N\left(p_{k+1}^{k} \circ{ }^{j} R \circ \bar{f}, X, x\right) d \mathcal{L}_{k} x \leqq \mathcal{F}_{k+1}^{k}\left[\bar{f}^{*}(X)\right] \\
& =L(\bar{f} \mid X) \leqq \sum_{i=1}^{k+1} \int_{E_{k}} N\left(p_{k+1}^{k} \circ{ }^{i} R \circ \bar{f}, X, x\right) d \mathcal{L}_{k} x \\
& =\sum_{i=1}^{k} \int_{X_{(i)}} T_{v=\text { inf } X(i)}^{\sup X(i)^{u}} f\left(u_{1}, \cdots, u_{i-1}, v, u_{i+1}, \cdots, u_{k}\right) d \mathcal{L}_{k-1} u+\mathcal{L}_{k}(X) .
\end{aligned}
$$

3.15 THEOREM. The following statements are equivalent:

(i) $f$ is $B V T$ on $X$.

(ii) $L(\bar{f} \mid X)<\infty$.

Proof. This statement is an immediate consequence of the preceding lemma.

3.16 THEOREM. If $f$ is $B V T$ on $X$ and $U$ is the subset of $X$ on which $\bar{f}$ is approximately differentiable, then

(i) $\mathcal{L}_{k}(X-U)=0$,

(ii) $\int_{Y} J \bar{f}(x) d \mathcal{L}_{k} x=\mathcal{F}_{k+1}^{k}\left[\bar{f}^{*}(Y \cap U)\right] \leqq \mathcal{F}_{k+1}^{k}\left[\bar{f}^{*}(Y)\right]<\infty$ whenever $Y$ is an $\mathcal{L}_{k}$ measurable subset of $X$.

Proof. Let $j$ be a positive integer no greater than $k$. Just as in Theorem 2.14 we can show that

$$
D_{j} f(x) \text { exists for } \mathcal{L}_{k} \text { almost all } x \text { in } X .
$$

Whence

$$
D_{i} \bar{f}(x) \text { exists for } \mathcal{L}_{k} \text { almost all } x \text { in } X,
$$

and Stepanoff's theorem (see [S, IX 12.2]) implies that $\mathcal{L}_{k}(X-U)=0$. We may apply $[\mathrm{F} 2,5.2]$, with the measure $\Phi$ replaced by $\mathcal{F}_{k+1}^{k}$ (see $[\mathrm{F} 5,5.10]$ ), and Theorem 3.15 to obtain

$$
\begin{aligned}
\int_{Y} J \bar{f}(x) d \mathcal{L}_{k} x & =\int_{Y \cap U} J \bar{f}(x) d \mathcal{L}_{k} x=\int_{E_{k}} N(\bar{f}, Y \cap U, y) d \mathcal{F}_{k+1}^{k} y \\
& =\mathcal{F}_{k+1}^{k}\left[\bar{f}^{*}(Y \cap U)\right] \leqq \mathcal{F}_{k+1}^{k}\left[\bar{f}^{*}(Y)\right]<\infty .
\end{aligned}
$$


3.17 Theorem. If $f$ is $B V T$ on $X$, then the following statements are equivalent:

(i) $\mathcal{F}_{k+1}^{k}\left[\bar{f}^{*}(V)\right]=0$ whenever $V \subset X$ and $\mathcal{L}_{k}(V)=0$,

(ii) $\int_{Y} J \bar{f}(x) d \mathcal{L}_{k} x=\mathcal{F}_{k+1}^{k}\left[\bar{f}^{*}(Y)\right]<\infty$ whenever $Y$ is an $\mathcal{L}_{k}$ measurable subset of $X$,

(iii) $\int_{X} J \bar{f}(x) d \mathcal{L}_{k} x=\mathcal{F}_{k+1}^{k}\left[\bar{f}^{*}(X)\right]<\infty$,

(iv) $f$ is $A C T$ on $X$.

Proof. Let $V \subset X$ and $\mathcal{L}_{k}(V)=0$. Select a Borel subset $A$ of $E_{k}$ so that

$$
V \subset A \subset X, \quad \mathcal{L}_{k}(A)=0 .
$$

The proof will be divided into five parts.

PART 1. (i) implies (ii).

Proof. Let $Y$ be an $\mathcal{L}_{k}$ measurable subset of $X$ and $U$ be the subset of $X$ on which $\bar{f}$ is approximately differentiable. Then

$$
\begin{aligned}
\mathcal{L}_{k}(Y-U) & =0, \\
\mathcal{F}_{k+1}^{k}\left[\bar{f}^{*}(Y-U)\right] & =0,
\end{aligned}
$$

and Theorem 3. 16 implies

$$
\begin{aligned}
\int_{Y} J \bar{f}(x) d \mathcal{L}_{k} x & =\mathcal{F}_{k+1}^{k}\left[\bar{f}^{*}(Y \cap U)\right]+\mathcal{F}_{k+1}^{k}\left[\bar{f}^{*}(Y-U)\right] \\
& =\mathcal{F}_{k+1}^{k}\left[\bar{f}^{*}(Y)\right]<\infty .
\end{aligned}
$$

PART 2. (ii) implies (iii).

PART 3. (iii) implies (i).

Proof. Let $\epsilon>0$. Choose on $X$ a non-negative continuous function $c$ and a number $M$ satisfying

$$
\int_{X}|J f(x)-c(x)| d \mathcal{L}_{k} x \leqq \epsilon, \quad M>\sup _{x \in X} c(x) .
$$

We can select an open set $G$ of $E_{k}$ for which

$$
V \subset G, \quad \mathcal{L}_{k}(G)<\epsilon \cdot M^{-1},
$$

and a grating of $k-1$ planes which defines such a family $\left\{X_{i} \mid i=1,2, \cdots\right\}$ of subsets of $E_{k}$ that

$$
\begin{array}{r}
V \subset \bigcup_{i=1}^{\infty} X_{i} \subset G \cap X, \quad X_{i} \text { is a } k \text { cell for } i=1,2, \cdots, \\
\text { interior } X_{i} \cap \text { interior } X_{j}=0 \quad \text { for } i \neq j .
\end{array}
$$

Then 


$$
\begin{aligned}
\mathcal{F}_{k+1}^{k}\left[\bar{f}^{*}(V)\right] & \leqq \mathcal{F}_{k+1}^{k}\left[\bar{f}^{*}\left(\bigcup_{i=1}^{\infty} X_{i}\right)\right] \leqq \sum_{i=1}^{\infty} \mathcal{F}_{k+1}^{k}\left[\bar{f}^{*}\left(X_{i}\right)\right] \\
& =\sum_{i=1}^{\infty} \int_{x_{i}} J \bar{f}(x) d \mathcal{L}_{k} x=\sum_{i=1}^{\infty} \int_{\text {interior } x_{i}} J \bar{f}(x) d \mathcal{L}_{k} x \\
& \leqq \int_{G \cap x} J \bar{f}(x) d \mathcal{L}_{k} x \leqq \int_{G \cap x} c(x) d \mathcal{L}_{k} x+\epsilon<2 \epsilon .
\end{aligned}
$$

Because of the arbitrary nature of $\epsilon$ the proof is complete.

PART 4. (ii) implies (iv).

Proof. Suppose $j$ is a positive integer no greater than $k$.

The truth of (ii) means

$$
\mathcal{F}_{k+1}^{k}\left[\bar{f}^{*}(A)\right]=0,
$$

and it follows from Lemma 3.12 and Theorem 2.15 that

$$
\begin{gathered}
\mathcal{L}_{k}\left[\left(p_{k+1}^{k} \circ{ }^{j} R \circ \bar{f}\right)^{*}(A)\right]=0, \quad \mathcal{L}_{k}\left[\left(p_{k+1}^{k} \circ{ }^{i} R \circ \bar{f}\right)^{*}(V)\right]=0, \\
p_{k+1}^{k} \circ{ }^{j} R \circ \bar{f} \text { is absolutely continuous on } X, \\
f \text { is } \operatorname{ACT}(j) \text { on } X .
\end{gathered}
$$

Consequently

$$
f \text { is ACT on } X \text {. }
$$

PART 5. (iv) implies (i).

Proof. Using Theorem 2.15 we know that

$f$ is $\operatorname{ACT}(i)$ on $X$, $p_{k+1}^{k} \circ{ }^{i} R \circ \bar{f}$ is absolutely continuous on $X$,

$$
\int_{E_{k}} N\left(p_{k+1}^{k} \circ{ }^{i} R \circ \bar{f}, A, x\right) d \mathcal{L}_{k} x=0,
$$

for $i=1,2, \cdots, k$. Recalling that

$$
\int_{E_{k}} N\left(p_{k+1}^{k} \circ{ }^{k+1} R \circ \bar{f}, A, x\right) d \mathcal{L}_{k} x=\mathcal{L}_{k}(A),
$$

we may conclude from Lemma 3.12 that

$$
\mathcal{F}_{k+1}^{h}\left[\bar{f}^{*}(A)\right]=0, \quad \mathcal{F}_{k+1}^{k}\left[\bar{f}^{*}(V)\right]=0 .
$$

3.18 REMARK. If

(i) $h$ is a continuous function on $X$ into $X$,

(ii) $\int_{E_{k}} N(h, X, x) d \mathcal{L}_{k} x<\infty$,

(iii) $f$ is $A C T$ on $X$, 
then $h$ is absolutely continuous on $X$ if and only if

$$
\mathcal{F}_{k+1}^{k}\left[(\bar{f} \circ h)^{*}(V)\right]=0 \text { whenever } V \subset X \text { and } \mathcal{L}_{k}(V)=0 \text {. }
$$

This is a consequence of Theorem 3.17.

3.19 REMARK. Using the notation of [F6] we may restate the results of Theorems 3.8, 3.16, and 3.17:

$$
\begin{aligned}
L(\bar{f} \mid X)<\infty \text { implies } & \\
\int_{X} J \bar{f}(x) d \mathcal{L}_{k} x \leqq L(\bar{f} \mid X) & =M^{* *}(\bar{f} \mid X)=S^{* *}(\bar{f} \mid X)=U^{* *}(\bar{f} \mid X) \\
& =V^{* *}(\bar{f} \mid X)=N^{* *}(\bar{f} \mid X),
\end{aligned}
$$

equality holding if and only if $f$ is ACT on $X$.

3.20 THEOREM. If for $x \in E_{k}$ and $r>0$

$$
C(x, r)=E_{k} \cap\{z|| z-x \mid \leqq r\},
$$

then

$$
\begin{gathered}
f \text { is BVT on } X \text { implies } \\
\lim _{r \rightarrow 0+} \frac{L[\bar{f} \mid C(x, r)]}{\alpha(k) r^{k}}=J \bar{f}(x) \quad \text { for } \mathcal{L}_{k} \text { almost all } x \in \text { interior } X .
\end{gathered}
$$

Proof. Letting Theorems 3.8 and 3.16 play the respective roles of Theorems 2.6 and 2.14, the proof is similar to that of Theorem 2.18 . In fact only the verification of the additivity of the singular part of the decomposition is different. For this the following general property of Carathéodory outer measures proves useful:

if $\phi$ is a Carathéodory outer measure, $A, B$, and $T$ are elements of domain $\phi$, and $A$ is $\phi$ measurable, then

$$
\phi(T \cap(A \cup B))+\phi(T \cap A \cap B)=\phi(T \cap A)+\phi(T \cap B) .
$$

\section{BIBLIOGRAPHY}

C. Caratheodory

[C] Über das lineare Mass von Punktmengen-eine Verallgemeinerung des Längenbegriffs, H. FEDERER Nachr. Ges. Wiss. Göttingen (1914) pp. 404-426.

[F1] Surface area. I, Trans. Amer. Math. Soc vol. 55 (1944) pp. 420-437.

[F2] Surface area. II, Trans. Amer. Math. Soc. vol. 55 (1944) pp. 438-456.

[F3] The Gauss-Green theorem, Trans. Amer. Math. Soc. vol. 58 (1945) pp. 44-76.

[F4] Coincidence functions and their integrals, Trans. Amer. Math. Soc. vol. 59 (1946) pp. 441-466.

[F5] The $(\phi, k)$ rectifiable subsets of $n$ space, Trans. Amer. Math. Soc. vol. 62 (1947) pp. 114-192. 
[F6] Hausdorff measure and Lebesgue area, Proc. Nat. Acad. Sci. U.S.A. vol. 37 (1951) pp. 90-94.

[F7] Measure and area, Bull. Amer. Math. Soc. vol. 58 (1952) pp. 306-378.

F. HAUSDORFF

[H] Dimension und äusseres Mass, Math. Ann. vol. 79 (1918) pp. 157-179.

P. V. REICHELDERFER

[R] On bounded variation and absolute continuity for parametric representation of continuous surfaces, Trans. Amer. Math. Soc. vol. 53 (1943) pp. 251-291.

S. SAKS

[S] Theory of the integral, Warsaw, 1937.

A. SARD

[SD] The equivalence of $n$-measure and Lebesgue measure in $E_{n}$, Bull. Amer. Math. Soc. vol. 49 (1943) pp. 758-759.

L. TONELLI

[T1] Sulla quadratura della superficie, Rendiconti Acc. Lincei (6) vol. $3_{1}$ (1926) pp. 357362, pp. 445-450, pp. 633-638, pp. 714-719.

[T2] Su un polinomio d'approssimazione e l'area di una superficie, Rendiconti Acc. Lincei A. WeIL (6) vol. 5 (1927) pp. 313-318.

[W] L'intégration dans les groupes topologiques et ses applications, Actualités Scientifiques et Industrielles, vol. 869, Paris, Hermann, 1938.

\section{BROWN UNIVERSITY,}

Providence, R. I. 